\title{
Fluid-Elastic Instability Tests on Parallel Triangular Tube Bundles with Different Mass Ratio Values under Increasing and Decreasing Flow Velocities
}

\author{
Xu Zhang, ${ }^{1,2,3}$ Bin Jiang, ${ }^{1,2,3}$ Luhong Zhang, ${ }^{1,3}$ and Xiaoming Xiao ${ }^{1,3}$ \\ ${ }^{1}$ School of Chemical Engineering and Technology, Tianjin University, Tianjin 300072, China \\ ${ }^{2}$ National Engineering Research Center for Distillation Technology, Tianjin 300072, China \\ ${ }^{3}$ Tianjin Collaborative Innovation Center of Chemical Science and Engineering, Tianjin 300072, China \\ Correspondence should be addressed to Xiaoming Xiao; xmxiao@tju.edu.cn
}

Received 26 May 2016; Revised 6 August 2016; Accepted 28 September 2016

Academic Editor: Marco Belloli

Copyright (c) 2016 Xu Zhang et al. This is an open access article distributed under the Creative Commons Attribution License, which permits unrestricted use, distribution, and reproduction in any medium, provided the original work is properly cited.

\begin{abstract}
To study the effects of increasing and decreasing flow velocities on the fluid-elastic instability of tube bundles, the responses of an elastically mounted tube in a rigid parallel triangular tube bundle with a pitch-to-diameter ratio of 1.67 were tested in a water tunnel subjected to crossflow. Aluminum and stainless steel tubes were tested, respectively. In the in-line and transverse directions, the amplitudes, power spectrum density functions, response frequencies, added mass coefficients, and other results were obtained and compared. Results show that the nonlinear hysteresis phenomenon occurred in both tube bundle vibrations. When the flow velocity is decreasing, the tubes which have been in the state of fluid-elastic instability can keep on this state for a certain flow velocity range. During this process, the response frequencies of the tubes will decrease. Furthermore, the response frequencies of the aluminum tube can decrease much more than those of the stainless steel tube. The fluid-elastic instability constants fitted for these experiments were obtained from experimental data. A deeper insight into the fluid-elastic instability of tube bundles was also obtained by synthesizing the results. This study is beneficial for designing and operating equipment with tube bundles inside, as well as for further research on the fluid-elastic instability of tube bundles.
\end{abstract}

\section{Introduction}

Flow-induced vibration remains a concern in designing tube-like structures, such as shell-and-tube heat exchangers, nuclear steam generators, and tubular reactors. Flowinduced vibration of tube bundles mainly contains fluidelastic instability, vortex shedding, turbulent buffeting, and acoustic resonance [1-4]. Among these characteristics, fluidelastic instability, which is a self-excited vibration, represents the most possible danger because of its potential for extremely large vibration amplitudes.

When the flow exceeds a certain critical velocity, fluidelastic instability occurs with the rapid increase in the amplitude of the tube bundle. Two mechanisms are commonly recognized as being responsible for fluid-elastic instability: damping mechanism $[5,6]$ and stiffness mechanism [7]. The tube bundle may be damaged by large-amplitude vibrations in a short time. Therefore, the mechanism of fluid-elastic instability should be studied for predicting and avoiding dangerous flow conditions in tube-like structures.

Over the past decades, numerous scholars have conducted many theoretical and experimental studies on fluidelastic instability. Connors [8] proposed the first criterion for fluid-elastic instability. Afterwards, many theoretical models for different conditions were provided in a series of papers by Pettigrew and Gorman [9], Païdoussis [10], and Weaver and Fitzpatrick [11]. Piteau et al. [12] recently studied a loosely supported tube in a rigid bundle subjected to single-phase flow through experiments and computations. Yan et al. [13] tested the shell side flow-induced vibration of a conical spiral tube array. Anderson et al. [14] modeled fluid-elastic instability including the boundary layer effect, in a square inline tube array. El Bouzidi and Hassan [15] investigated time lag causing fluid-elastic instability in tube arrays. 


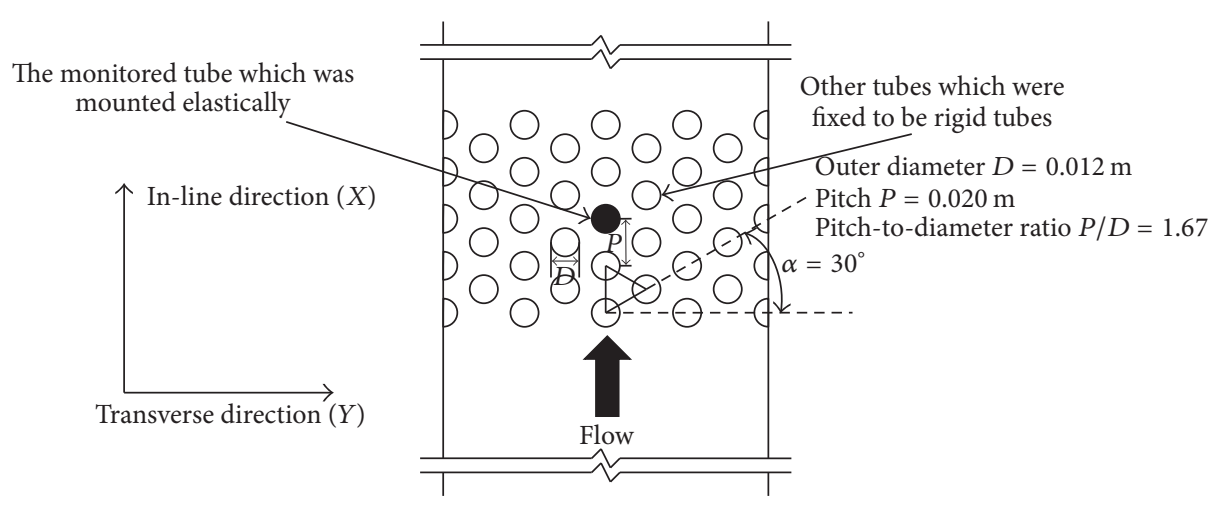

FIGURE 1: Schematic of the tube bundle installed in the test section of the experimental apparatus.

The operation of tube-like structures involves an increase and decrease in flow velocity. While, many scholars often only pay attention to the effort of increasing flow velocity on the fluid-elastic instability of tube bundles, neglecting the effort of decreasing flow velocity on tube bundles that are in the state of fluid-elastic instability. The study of the effort of decreasing flow velocity on tube bundles in the state of fluidelastic instability is important in industrial applications in which operational cost must be reduced.

With this background, under both increasing and decreasing crossflow velocities, experiments were conducted in a water tunnel containing parallel triangular tube bundles in which two different tube masses were used, respectively. The pitch-to-diameter ratio $(P / D)$ was maintained at 1.67. In the in-line and transverse directions, the amplitudes, power spectrum density functions, response frequencies, added mass coefficients, and other results were obtained and compared. Deeper insight into the fluid-elastic instability of tube bundles was also obtained by synthesizing the results. This study is beneficial for designing and operating equipment with tube bundles inside, as well as for further research on the fluidelastic instability of tube bundles.

\section{Experimental Details}

2.1. Experimental Apparatus and Instrumentation. Figure 1 shows the test section, which is widely used in flow-induced vibration research on tube bundles [16-21]. The working fluid was water, which was pumped through a tank, an electromagnetic flowmeter, two valves, and flowed past steady flow plates for stabilizing the incoming flow before entering the test section. The average turbulence intensity over the flow velocity range measured 10 diameters upstream of the tube bundle was less than $1.2 \%$, and the velocity distribution was uniform within $1 \%$ in the test section, excluding the boundary layer. The measuring range of the electromagnetic flowmeter was $0-200 \mathrm{~m}^{3} / \mathrm{h}$. The head and measuring range of the centrifugal pump were $20 \mathrm{~m}$ and $0-65 \mathrm{~m}^{3} / \mathrm{h}$, respectively.

The test section, which was made of stainless steel, had internal dimensions of $0.140 \mathrm{~m} \times 0.140 \mathrm{~m}$. The tube bundle consisted of a bundle of tubes with an outer diameter of
$0.012 \mathrm{~m}$, an inner diameter of $0.010 \mathrm{~m}$, and a length of $0.130 \mathrm{~m}$. The tube bundle was parallel triangular with a pitch-todiameter ratio of $P / D=1.67$. There were 9 rows, with alternately 4 or 3 and two-half tubes per row, which provided a uniform flow profile. The mass of each tube in the bundle could be changed by replacing it with another tube of a different material. During the whole experiments, two kinds of tubes were used, namely, aluminum and stainless steel tubes.

Fluid-elastic instability of tube bundles is affected by many parameters, such that a full model with a fully flexible tube bundle is highly complex and requires a large number of measured empirical coefficients $[5,6]$. Therefore, for a basic research to enhance insights into the physics of fluidelastic instability it is necessary to reduce the problem complexity and the amount of empirical coefficients required. An ordinary simplified method is to use a single elastically mounted tube in a rigid bundle to study the phenomenon. This method has been verified as feasible by Lever and Weaver [22, 23]. Many studies have been conducted on this basis, such as those performed by Price and Païdoussis [24], Marn and Catton [25], and Harran [26]. For simplification, the monitored tube was elastically mounted in the center of the tube bundle, whereas other tubes were fixed at the channel walls of the test section with bolts at two ends to be rigid tubes during the whole experiments.

As shown in Figure 2, the monitored tube was mounted elastically, which was equipped with two stainless steel piano lines, two screw tubes, two screw nuts, two springs, and two pins. One endpoint of every stainless steel piano line was fixed to the monitored tube, and the other endpoint was fixed to a pin. The spring was mounted between the screw nut and pin. To adjust the natural frequency of the vibration system, screw nuts were used to adjust the springs to tighten or loosen the stainless steel piano lines. The displacement mechanism of the monitored tube was derived from Joo and Dhir [27], Mitra [28], and Cagney and Balabani [29]. The monitored tube could vibrate in both the in-line and transverse directions simultaneously.

Acceleration transducers with sensitivity of $1.71 \mathrm{pC} / \mathrm{g}$ and frequency response of $1 \mathrm{~Hz}-20 \mathrm{kHz}$ were chosen to obtain the displacement signals directly and simultaneously [16, 30-32]. 


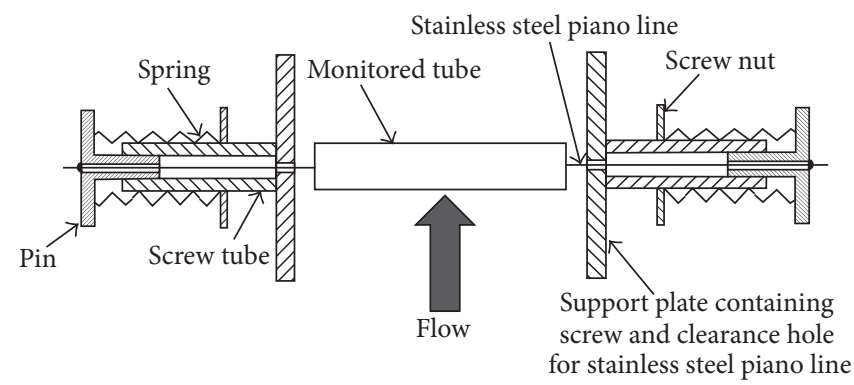

FIgURE 2: Schematic of the installation of the monitored tube.

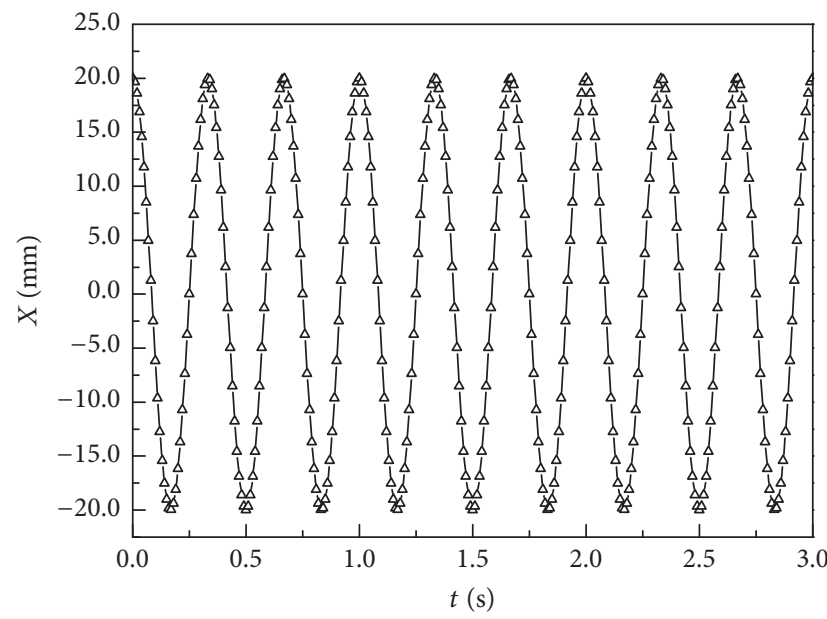

(a)

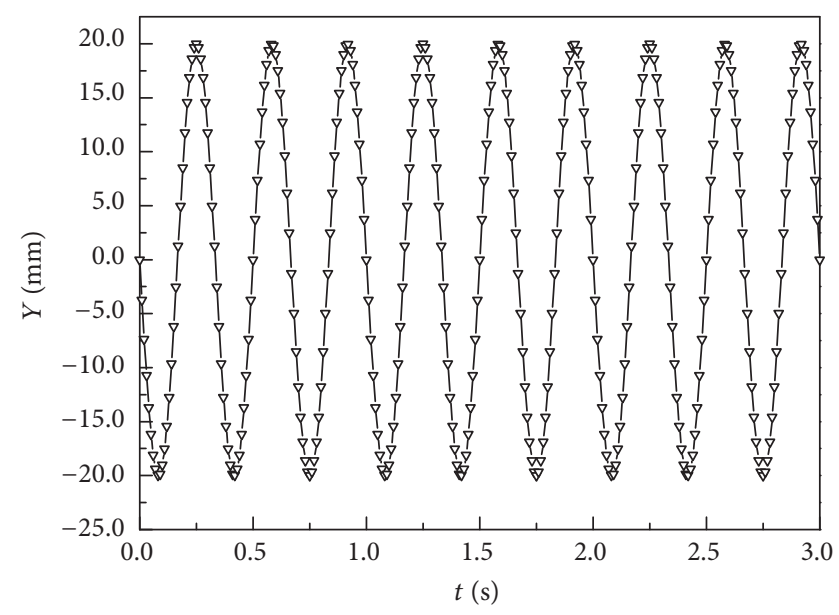

(b)

FiguRE 3: Typical time-history curves at the time for calibration of two acceleration transducers. (a) In-line direction and (b) transverse direction.

The output signal was calibrated to have a linear relationship with the input signal. Two acceleration transducers installed inside the monitored tube at midspan were treated as part of the monitored tube throughout the whole experiments. Their orientations were adjusted such that they were sensitive to tube vibrations only in the in-line $(X)$ and transverse $(Y)$ directions. The output signals of the two acceleration transducers were collected by a dynamic signal collector with a 24-bit analog to digital converter. The dynamic signal collector could transform the output signals to voltage signals and then integrate the voltage signal twice to provide a displacement signal. All data were transmitted to a personal computer for data storage and further processing. All signals were high-pass filtered in postprocessing at $1.2 \mathrm{~Hz}$ to prevent a "blow-up" that commonly occurs because of low frequency noises when converting acceleration to displacement. The cut-off frequency of $1.2 \mathrm{~Hz}$ was significantly lower than the response frequencies obtained from the whole experiments.

Before the experiments, the output signals of the whole data acquisition system were calibrated just as Lin and Yu [16] have done. A monitored tube with two acceleration transducers inside was given a circular motion by a turntable. The radius of the circular motion could be changed by altering the position of the tube. The results corresponding to the aluminum tube were the same as those corresponding to the stainless steel tube. The typical time-history curves and calibration curves of two acceleration transducers are shown in Figures 3 and 4 . The amplitudes of the sinusoidal output signals for the in-line and transverse directions were recorded and then plotted against the standard vibration amplitude, which was the radius of circular motion. The calibration curves can be determined as

$$
\begin{aligned}
& A=1.000 B+0.0536 \text { in the in-line direction, } \\
& A=1.000 B+0.0738 \text { in the transverse direction, }
\end{aligned}
$$

where $A$ represents the standard vibration amplitude and $B$ represents the measured vibration amplitude obtained by the whole data acquisition system. As shown in Figure 4, measured amplitude signals were all linear to the standard amplitude signals, so the measured amplitudes could represent the vibration amplitudes linearly in the whole experiments.

Prior to the water tunnel tests, the natural frequencies and damping ratios of the monitored tubes were measured by using the logarithmic decrement technique. The data 


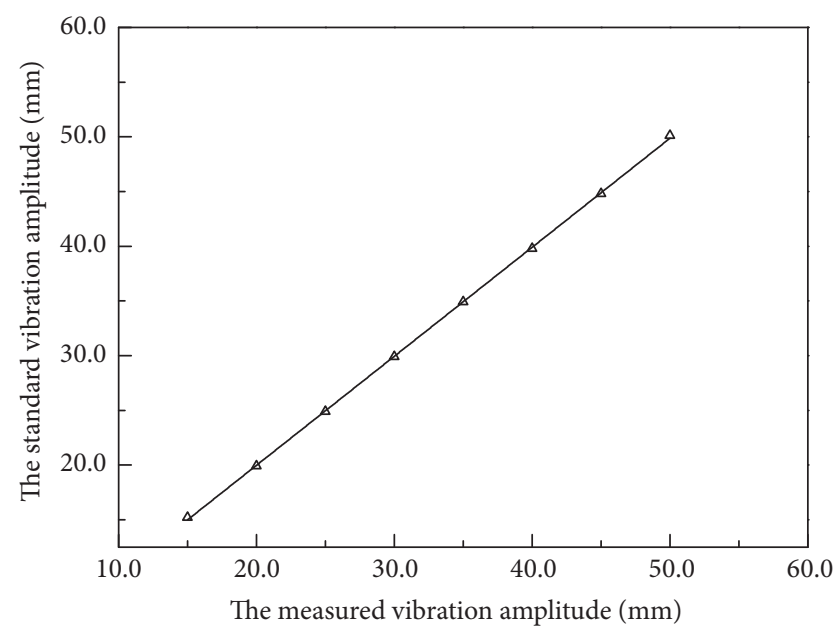

(a)

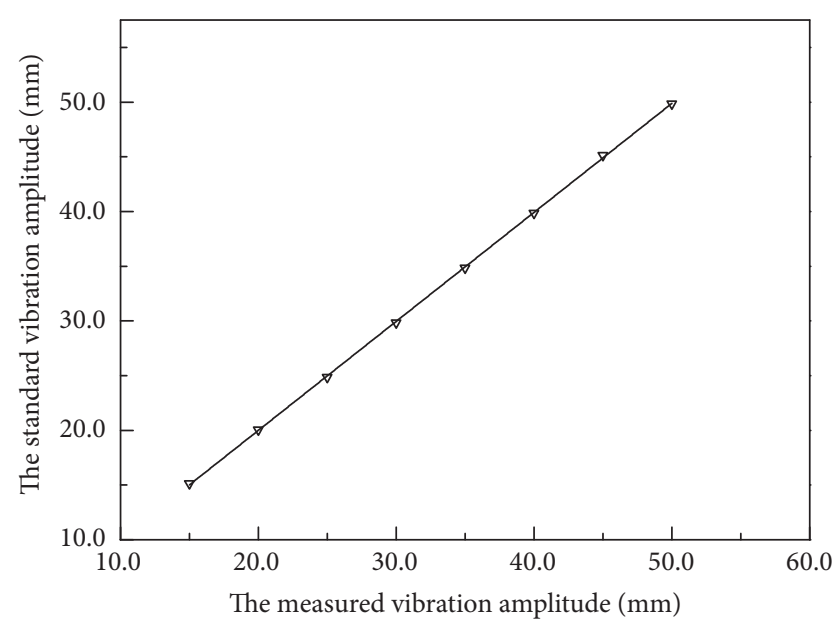

(b)

FIGURE 4: Fitted curves for calibration of two acceleration transducers. (a) In-line direction and (b) transverse direction.

TABLE 1: Monitored tubes data without flow.

\begin{tabular}{|c|c|c|}
\hline & Aluminum tube & Stainless steel tube \\
\hline Natural frequency in air in the in-line direction, $f_{\text {na-x }}(\mathrm{Hz})$ & 10.547 & 9.668 \\
\hline Natural frequency in air in the transverse direction, $f_{\text {na }-y}(\mathrm{~Hz})$ & 10.547 & 9.668 \\
\hline Damping ratio in air in the in-line direction, $\xi_{x a}$ & $0.829 \%$ & $0.813 \%$ \\
\hline Damping ratio in air in the transverse direction, $\xi_{y a}$ & $0.845 \%$ & $0.821 \%$ \\
\hline Natural frequency in still water in the in-line direction, $f_{n w_{-} x}(\mathrm{~Hz})$ & 8.105 & 8.301 \\
\hline Natural frequency in still water in the transverse direction, $f_{n w_{-} y}(\mathrm{~Hz})$ & 8.105 & 8.301 \\
\hline Damping ratio in still water in the in-line direction, $\xi_{x w}$ & $5.121 \%$ & $3.734 \%$ \\
\hline Damping ratio in still water in the transverse direction, $\xi_{y w}$ & $5.162 \%$ & $3.816 \%$ \\
\hline Structural mass (including acceleration transducers inside), $M(\mathrm{~kg})$ & 0.02213 & 0.04619 \\
\hline Oscillating mass of the system, $m_{t}(\mathrm{~kg})$ & 0.03680 & 0.06087 \\
\hline Mass per unit length of the tube, $m(\mathrm{~kg} / \mathrm{m})$ & 0.2831 & 0.4682 \\
\hline Mass ratio $m^{*}$ & 2.508 & 4.147 \\
\hline
\end{tabular}

are summarized in Table 1. The time histories of the free vibrations of the monitored tubes are shown in Figures 5 and 6 . The mass per unit length of the tube $m$ and the mass ratio $m^{*}$ were calculated by using the following equations [33]:

$$
\begin{aligned}
m_{a} & =c_{a} m_{d}, \\
m_{d} & =\frac{\pi}{4} \rho D^{2} L, \\
m & =\frac{M+m_{a}}{L}, \\
m^{*} & =\frac{M+m_{a}}{m_{d}},
\end{aligned}
$$

where $m_{a}$ is the added mass, $c_{a}$ is the added mass coefficient, $m_{d}$ is the mass of the displaced fluid, $\rho$ is the density of the fluid, $D$ is the outer diameter of the tube, and $L$ is the length of the tube. Khalak and Williamson [34] demonstrated that the added mass coefficient $c_{a}$ can be thought to be 1 in still water.

2.2. Experimental Procedure. The freestream velocity in the test section was calculated using the following equation:

$$
U_{\infty}=\frac{V}{3600 a b}
$$

where $U_{\infty}$ is the freestream velocity in the test section with a unit of " $\mathrm{m} / \mathrm{s}$ ", $V$ is the flow rate with a unit of " $\mathrm{m}^{3} / \mathrm{h}$," $a$ is the length of the cross section of the test section (equal to $0.140 \mathrm{~m}$ ), and $b$ is the width of the cross section of the test section (equal to $0.140 \mathrm{~m}$ ).

In the beginning of the experiment, the flow rate initially increased stepwise until obvious fluid-elastic instability occurred. Subsequently, the flow rate decreased to zero stepwise in the same way. The maximum flow rates in experiments were $24.115 \mathrm{~m}^{3} / \mathrm{h}$ for the aluminum tube bundle and 


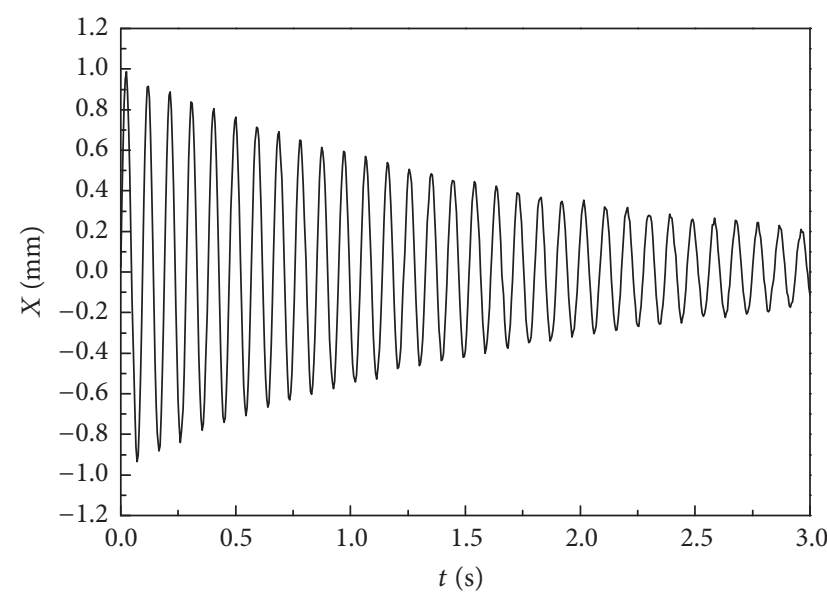

(a)

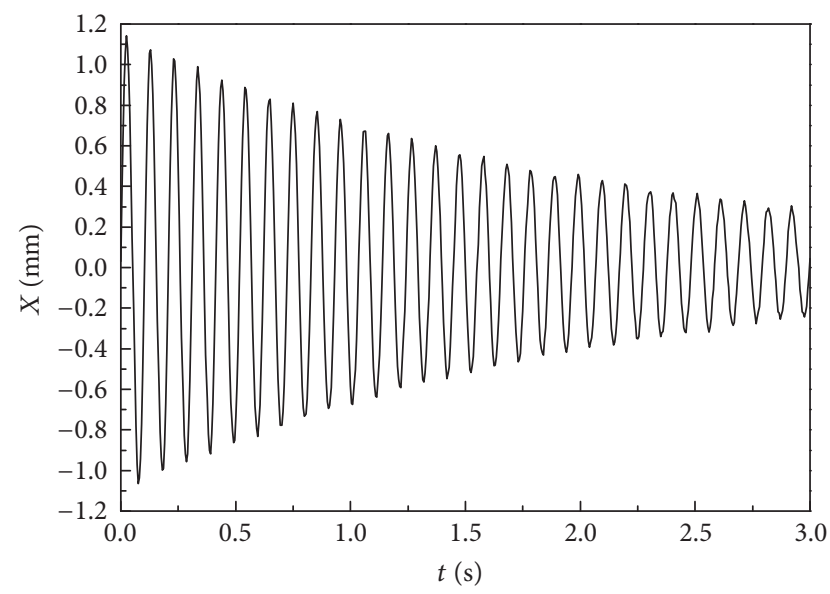

(c)

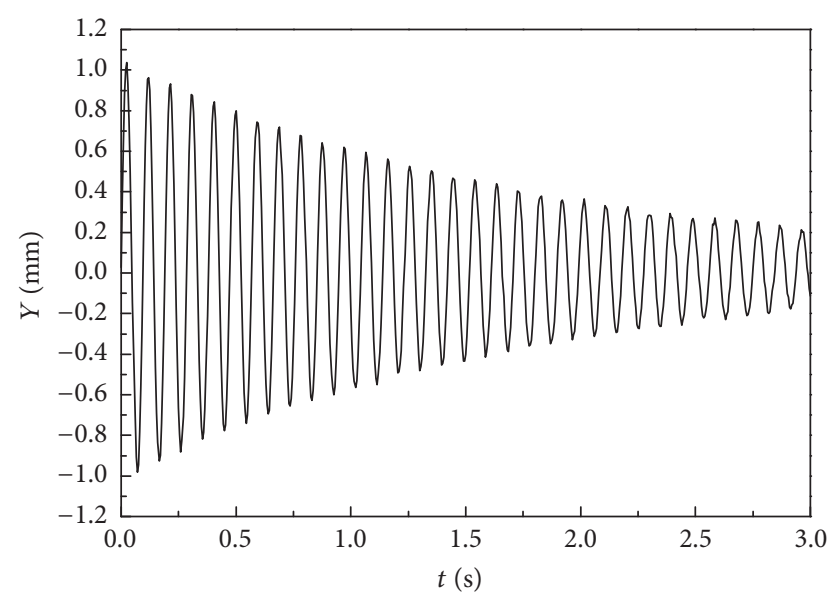

(b)

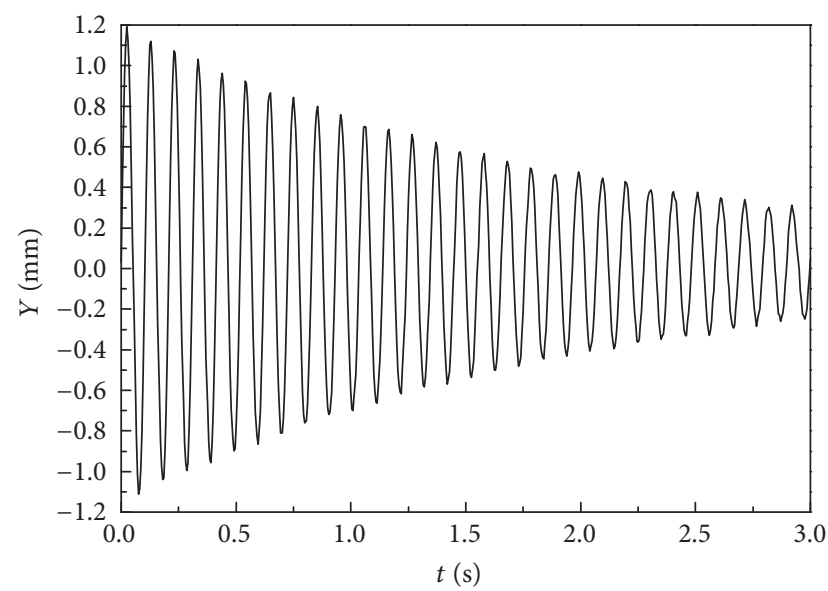

(d)

Figure 5: Time histories of the free vibrations of the monitored tubes in air. (a) Aluminum tube in the in-line direction, (b) aluminum tube in the transverse direction, (c) stainless steel tube in the in-line direction, and (d) stainless steel tube in the transverse direction.

$34.170 \mathrm{~m}^{3} / \mathrm{h}$ for the stainless steel tube bundle, which resulted in the maximum average water freestream velocities in the test section to reach 0.342 and $0.484 \mathrm{~m} / \mathrm{s}$, respectively. The corresponding maximum Reynolds numbers based on the maximum freestream velocities and the outer diameter of the experimental tubes were $0.407 \times 10^{4}$ and $0.577 \times 10^{4}$, respectively. Measurements were made after reaching a steady state for each flow rate. At each given flow velocity, the in-line and transverse displacement signals were digitized simultaneously at a sampling frequency of $200 \mathrm{~Hz}$. The frequency spectra and response amplitudes recorded were the result of 64 sample averages which was found adequate to insure excellent repeatability. MATLAB software was used for data analyses.

\section{Results and Discussion}

3.1. Response Amplitude. The response amplitude curves of the two kinds of monitored tubes are shown in Figure 7. To characterize the magnitude of tube vibrations, the rootmean-square tube amplitude expressed as a percentage of the tube diameter was plotted against reduced pitch flow velocity $U^{*}$ obtained from the following equations:

$$
\begin{aligned}
U_{P} & =\frac{P}{P-D} U_{\infty}, \\
U^{*} & =\frac{U_{P}}{f_{n} D},
\end{aligned}
$$

where $U_{\infty}$ is the freestream velocity in front of the tube bundle in the test section, $P$ is the pitch of the tube bundle, $D$ is the outer diameter of the tube, $U_{p}$ is the pitch flow velocity, and $f_{n}$ is the natural frequency of the aluminum tube or the stainless steel tube in still water in the in-line or transverse direction. During the experiments, the Reynolds number Re was simply proportional to the reduced pitch flow velocity $U^{*}$.

As shown in Figure 7, regardless of the direction (in-line or transverse), the trend of the response amplitude curves corresponding to the aluminum tube vibrations is similar to the trend of the response amplitude curves corresponding to the stainless steel tube vibrations with increasing flow 


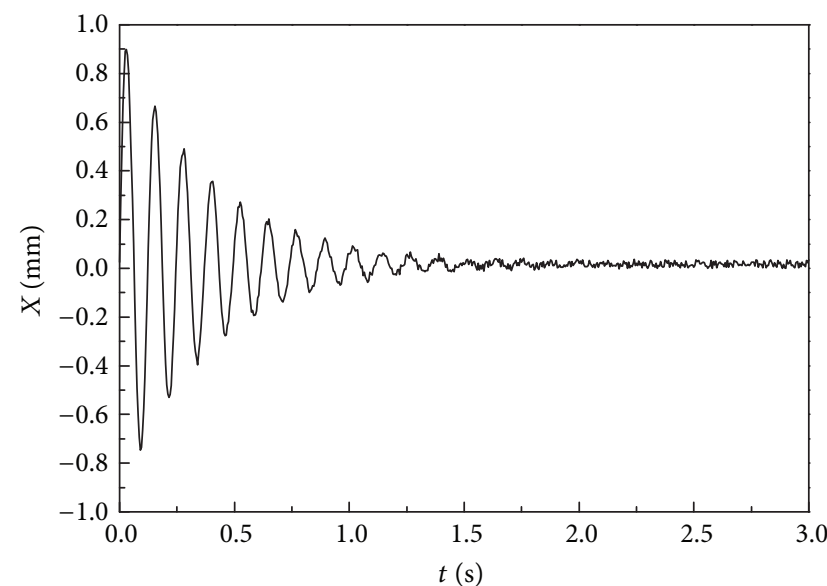

(a)

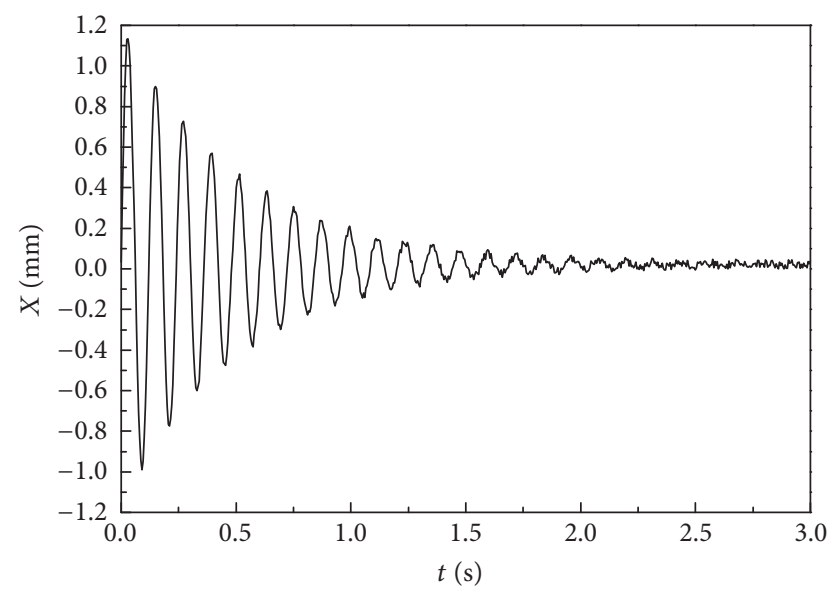

(c)

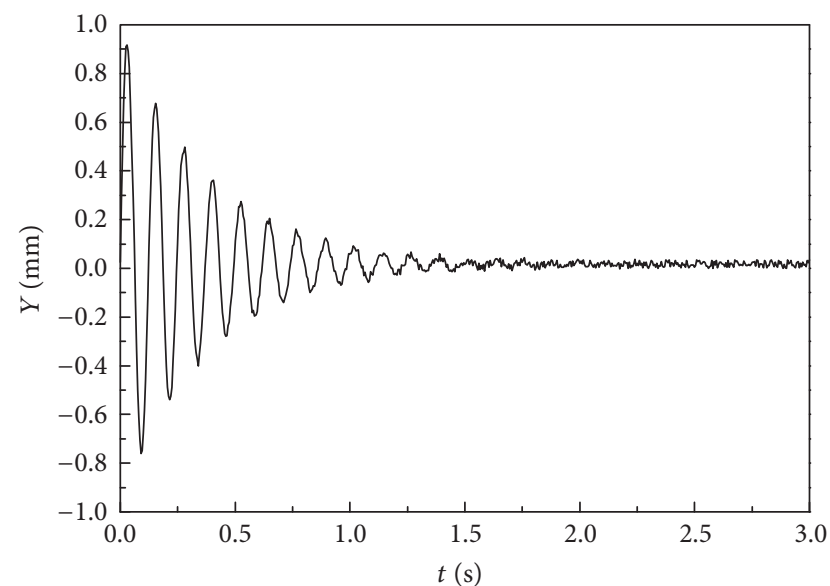

(b)

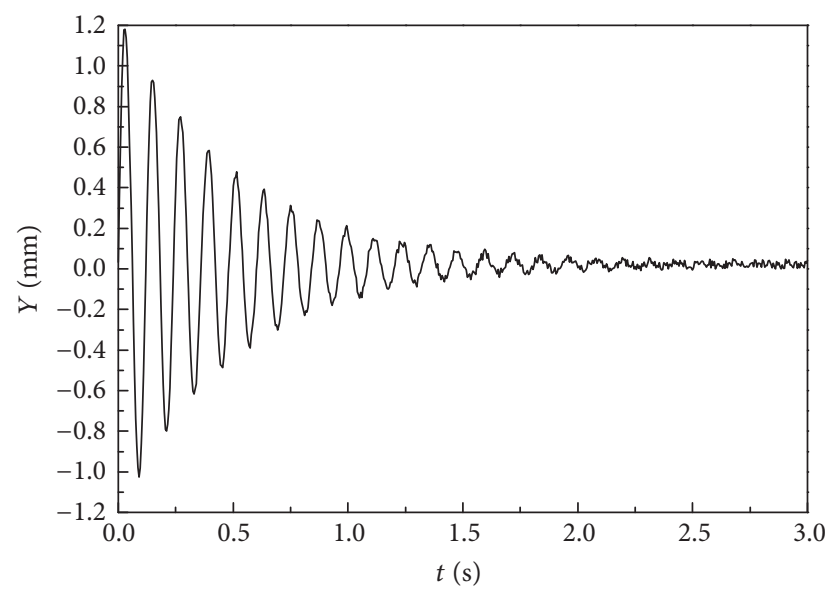

(d)

FIGURE 6: Time histories of the free vibrations of the monitored tubes in water. (a) Aluminum tube in the in-line direction, (b) aluminum tube in the transverse direction, (c) stainless steel tube in the in-line direction, and (d) stainless steel tube in the transverse direction.

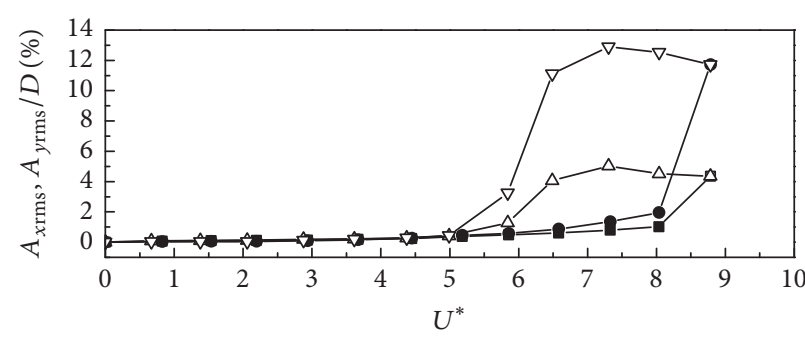

$$
\begin{array}{ll}
\text { In-line direction: } & \text { Transverse direction: } \\
-\square-\text { Increasing } U^{*} & -\bullet-\text { Increasing } U^{*} \\
-\triangle-\text { Decreasing } U^{*} & -\nabla-\text { Decreasing } U^{*}
\end{array}
$$

(a)

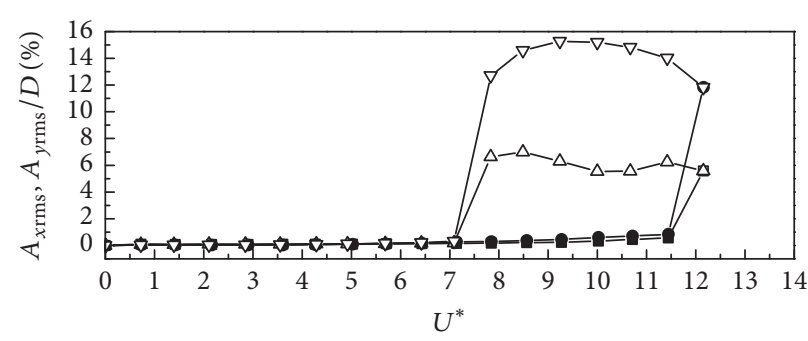

$$
\begin{array}{ll}
\text { In-line direction: } & \text { Transverse direction: } \\
-\square-\text { Increasing } U^{*} & -\bullet-\text { Increasing } U^{*} \\
-\triangle-\text { Decreasing } U^{*} & -\nabla-\text { Decreasing } U^{*}
\end{array}
$$

(b)

Figure 7: Root-mean-square response amplitudes. (a) Aluminum tube and (b) stainless steel tube.

velocity. The response amplitudes initially increase with $U^{*}$ in a very low degree, as the $U^{*}$ values are below two critical values $\left(U^{*} \approx 8.036\right.$ and $U^{*} \approx 11.439$ for the aluminum tube bundle and stainless steel tube bundle, resp.). These feeble vibrations can be contributed by turbulent buffeting with a broad frequency spectrum. As soon as the pitch flow velocities exceed the critical pitch flow velocities, the response amplitudes of the aluminum tube and stainless steel tube increase drastically in both the in-line and transverse directions. It indicates that fluid-elastic instability occurred. The 
serious vibration in the fluid-elastic instability phenomenon can be explained as follows. Flow can transport energy to the tube. When the flow velocity is above the critical flow velocity, the fluid has adequate energy to surmount the damping of the tube to make it vibrate with large amplitude at its natural frequency. Subsequently, fluid-elastic instability occurs.

After the tubes were in the state of fluid-elastic instability, the flow velocities began to decrease. While, as shown in Figure 7, the response amplitudes of the aluminum tube and stainless steel tube do not decrease immediately in either the in-line or transverse direction. By contrast, regardless of the direction (in-line or transverse), the response amplitude values of the aluminum tube and stainless steel tube slightly fluctuate around their maximum values in certain $U^{*}$ ranges, namely, $6.490 \leq U^{*} \leq 8.785$ for the aluminum tube bundle and $7.832 \leq U^{*} \leq 12.154$ for the stainless steel tube bundle. As the flow velocities further decrease, the response amplitudes of the aluminum tube and stainless steel tube significantly decrease in both the in-line and transverse directions. When $U^{*}$ decreases from 5.843 to 4.991 in the case of the aluminum tube and from 7.832 to 7.089 in the case of the stainless steel tube, the amplitudes drop a lot suddenly to nearly zero. This finding indicates that the fluid-elastic instability phenomenon ended. Thereafter, the amplitudes slightly decrease with the flow velocity, which suggests the recurrence of turbulent buffeting. Therefore, $U^{*} \approx 4.991$ and $U^{*} \approx 7.089$ were the critical reduced pitch flow velocities of the aluminum tube and stainless steel tube, respectively, for fluid-elastic instability under decreasing flow velocities. During fluid-elastic instability, the response amplitude curves obtained from the transverse vibrations are all above those obtained from the in-line vibrations under both increasing and decreasing $U^{*}$. Figure 7 also shows that the stainless steel tube exhibited higher peak amplitudes than the aluminum tube did during fluid-elastic instability when the flow velocity decreased. It should be caused by the reason that the damping ratio values of the monitored stainless steel tube in still water were lower than those of the monitored aluminum tube in still water, which has been shown in Table 1. This phenomenon will be investigated deeply in the future. According to the trend of the response amplitude curves shown in Figure 7, when the tubes are in the state of fluid-elastic instability, the curves corresponding to decreasing $U^{*}$ all gravely deviate from those corresponding to increasing $U^{*}$. Therefore, nonlinear hysteresis occurred, and the fluid-elastic instability phenomenon is also a nonlinear phenomenon. By this reason, when the fluid-elastic instability phenomenon has occurred in tube-like structures, the flow velocity should be reduced lower than the critical flow velocity corresponding to the case of increasing flow velocity for eliminating the fluidelastic instability phenomenon, resulting from the nonlinear hysteresis phenomenon.

3.2. Spectrum of Tube Vibration. To gain further insight into the vibration characteristics, a series of power spectrum density functions of the dimensionless vibration displacements of the monitored tubes have been obtained. Figures 8 and 9 show the power spectrum density functions of the aluminum tube vibrations with the increasing and decreasing
$U^{*}$, respectively. Figures 10 and 11 show the power spectrum density functions of the stainless steel tube vibrations with the increasing and decreasing $U^{*}$, respectively. As shown in Figures 8 and 10, the frequency spectra of both tubes are broadband in the $U^{*}$ ranges which are below the critical ones corresponding to the case of increasing $U^{*}$ for fluidelastic instability, resulting from the fact that the two kinds of monitored tubes are all in the state of turbulent buffeting. The broadband spectra reflect the random nature of the tube vibrations caused by the high flow turbulence in the tube bundles. After $U^{*}$ exceed the critical ones, the spectra immediately display clear sharp peaks, which indicated that the fluid-elastic instability phenomenon occurred. As shown in the cases of $U^{*} \approx 8.785$ in Figure 8 and $U^{*} \approx$ 12.154 in Figure 10, when the tube is in the state of fluidelastic instability, only one dominant peak is observed in the transverse vibration, whereas two prominent peaks are found in the in-line vibration. According to Franzini et al. [35], when the tube is in the state of fluid-elastic instability, the second prominent peak in each power spectral density function obtained from the in-line vibration is a subharmonic peak. Among both tube vibrations, the frequencies corresponding to the dominant peaks in the transverse vibrations and the frequencies corresponding to the first prominent peaks in the in-line vibrations are the natural frequencies of the monitored tubes. While, in the in-line vibrations, the frequency corresponding to the subharmonic peak is nearly twice as high as that corresponding to the first peak in the cases of both tubes. Thus, the monitored tubes are also vibrating in the resonance state and absorbing energy at their natural frequencies and the frequencies that are multiples of their natural frequencies.

As shown in Figures 9 and 11, when the flow velocities decrease, the clear sharp peaks do not disappear immediately. They are observed in certain $U^{*}$ ranges, which is consistent with Figure 7 . The frequencies corresponding to the sharp peaks fluctuate within a very narrow range. This indicates that the resonance state found in the fluid-elastic instability phenomenon can sustain in a certain flow velocity range in the case of decreasing flow velocity. This finding can further explain the nonlinear hysteresis phenomenon found in Figure 7. As soon as $U^{*}$ decrease to the critical values corresponding to the case of decreasing flow velocities, the spectra become broadband, reflecting that the monitored tubes have been in the state of turbulent buffeting again.

3.3. Response Frequency. To analyze the difference between the instability behaviors corresponding to increasing $U^{*}$ and those corresponding to decreasing $U^{*}$ furtherly, the in-line and transverse dominant frequencies (i.e., the frequency with the maximum amplitude in a power spectrum density function) of both tube vibrations in the $U^{*}$ ranges, where the response amplitude curves corresponding to increasing $U^{*}$ gravely deviate from those corresponding to decreasing $U^{*}$ in Figure 7, have been summarized and shown in Figure 12 in the form of ratio values to their natural frequencies in still water.

As shown in Figure 12, when the flow velocities increase, the dominant frequencies are all approaching the natural 

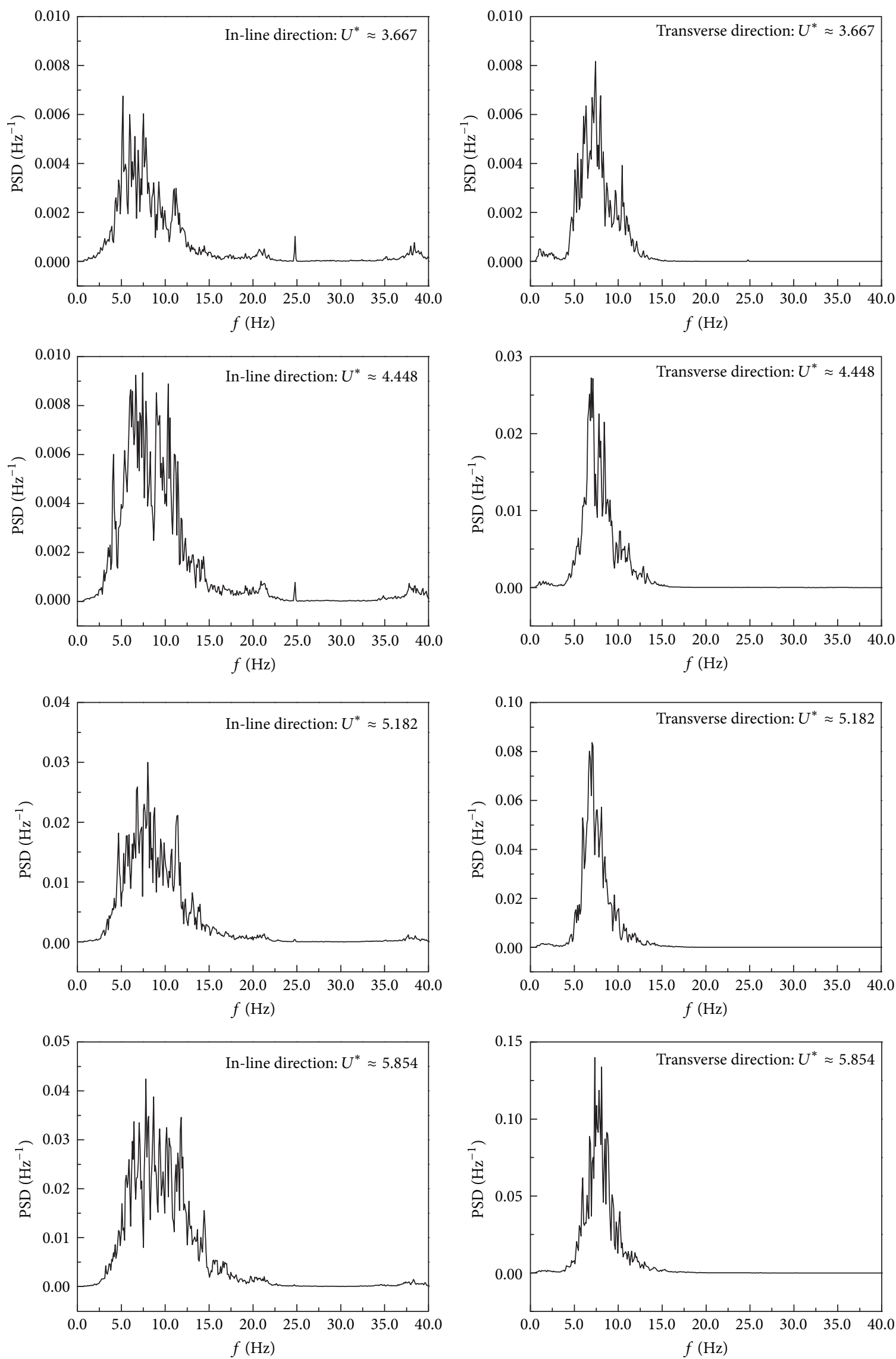

FIGURe 8: Continued. 

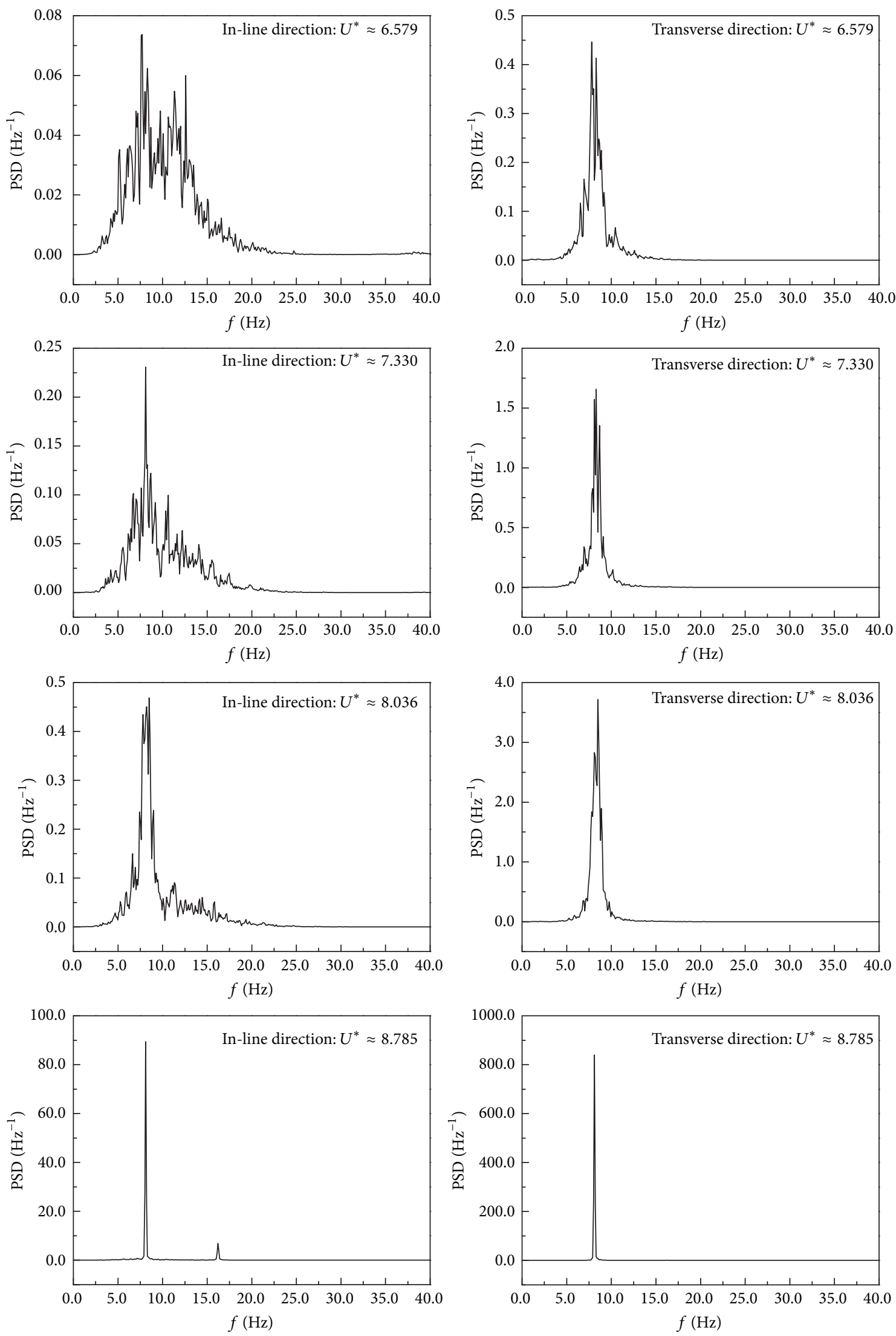

FIGURE 8: Power spectrum density functions of aluminum tube vibrations with the increasing of flow velocity. 

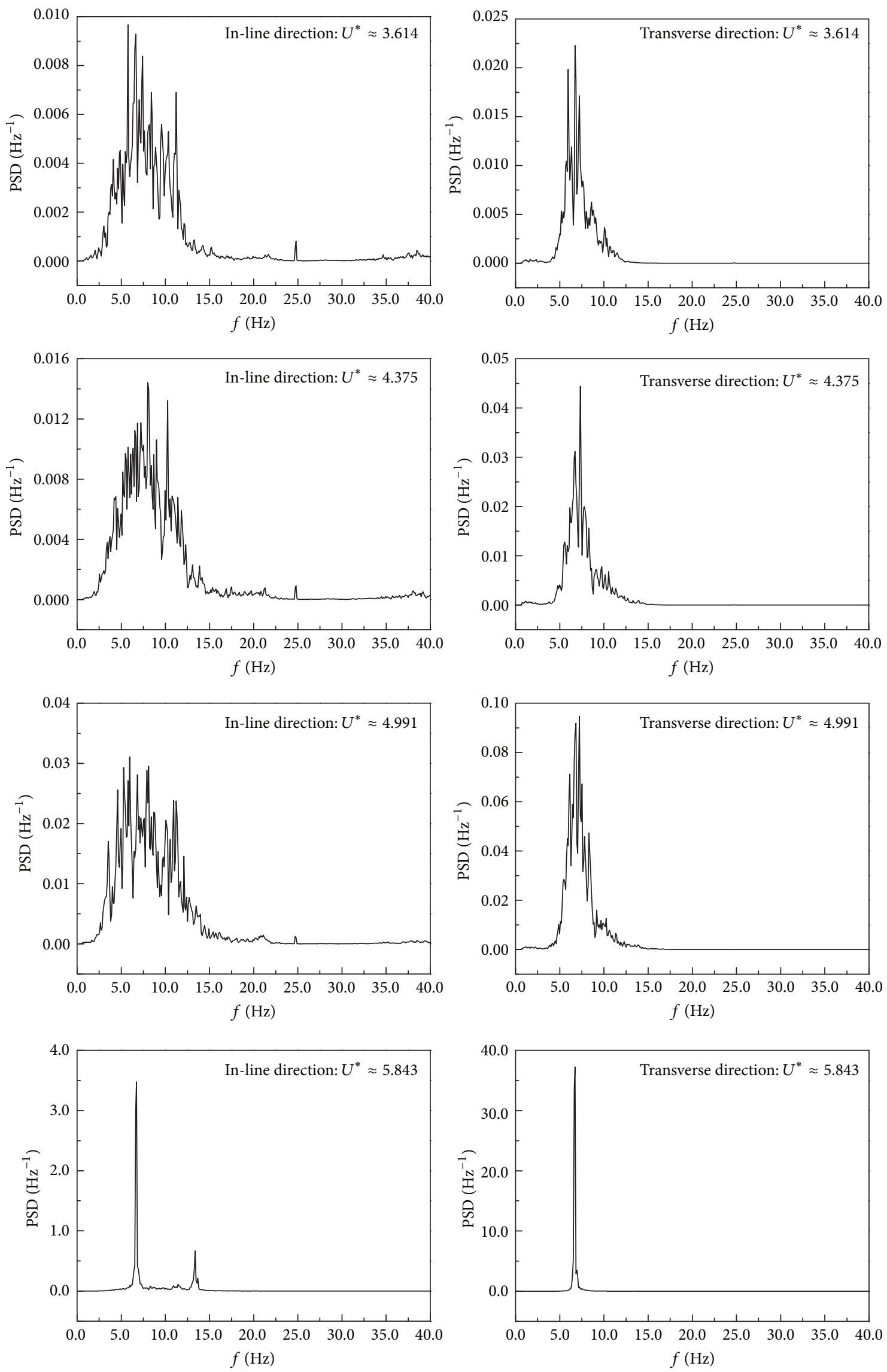

FIgure 9: Continued. 

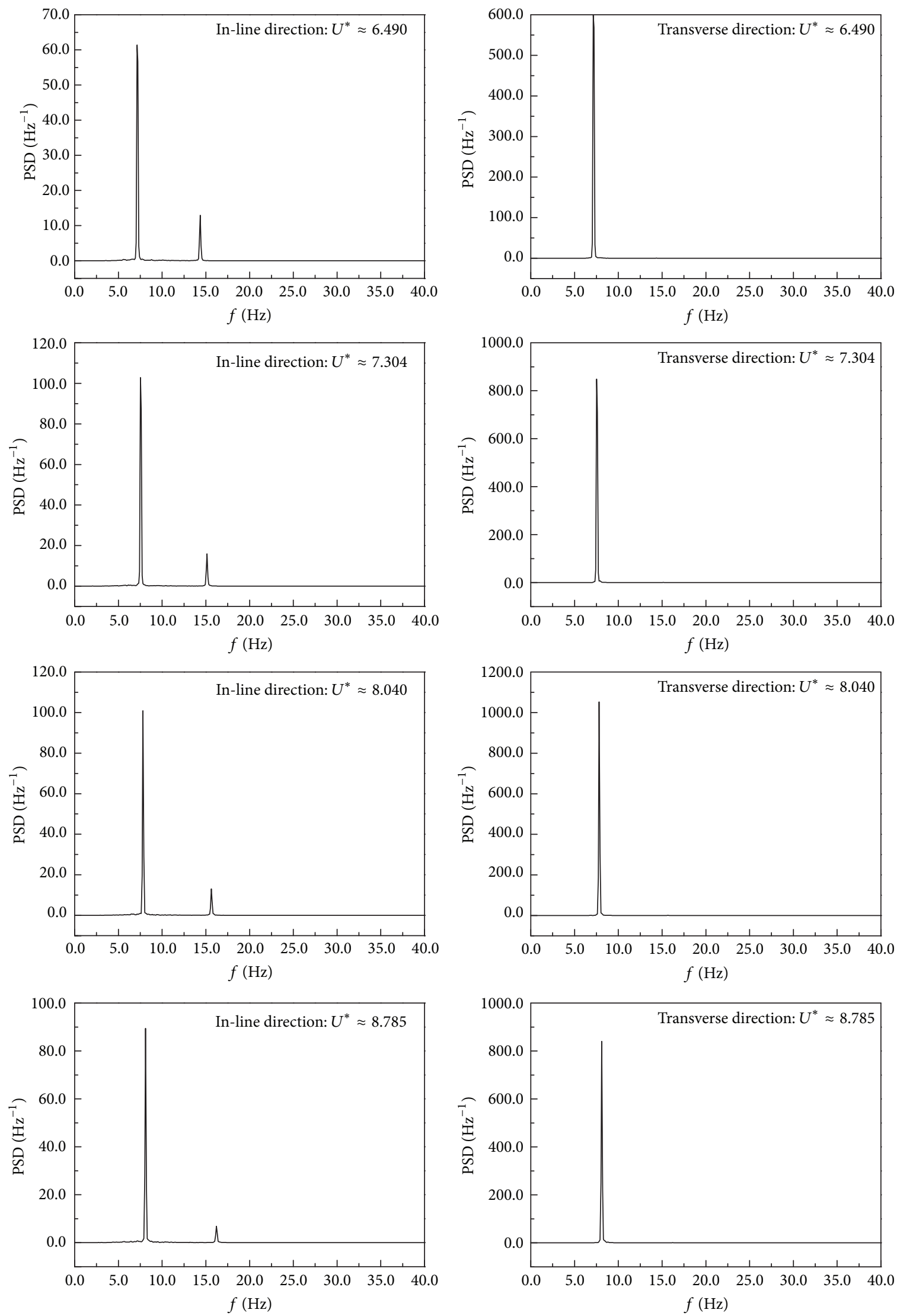

Figure 9: Power spectrum density functions of aluminum tube vibrations with the decreasing of flow velocity. 

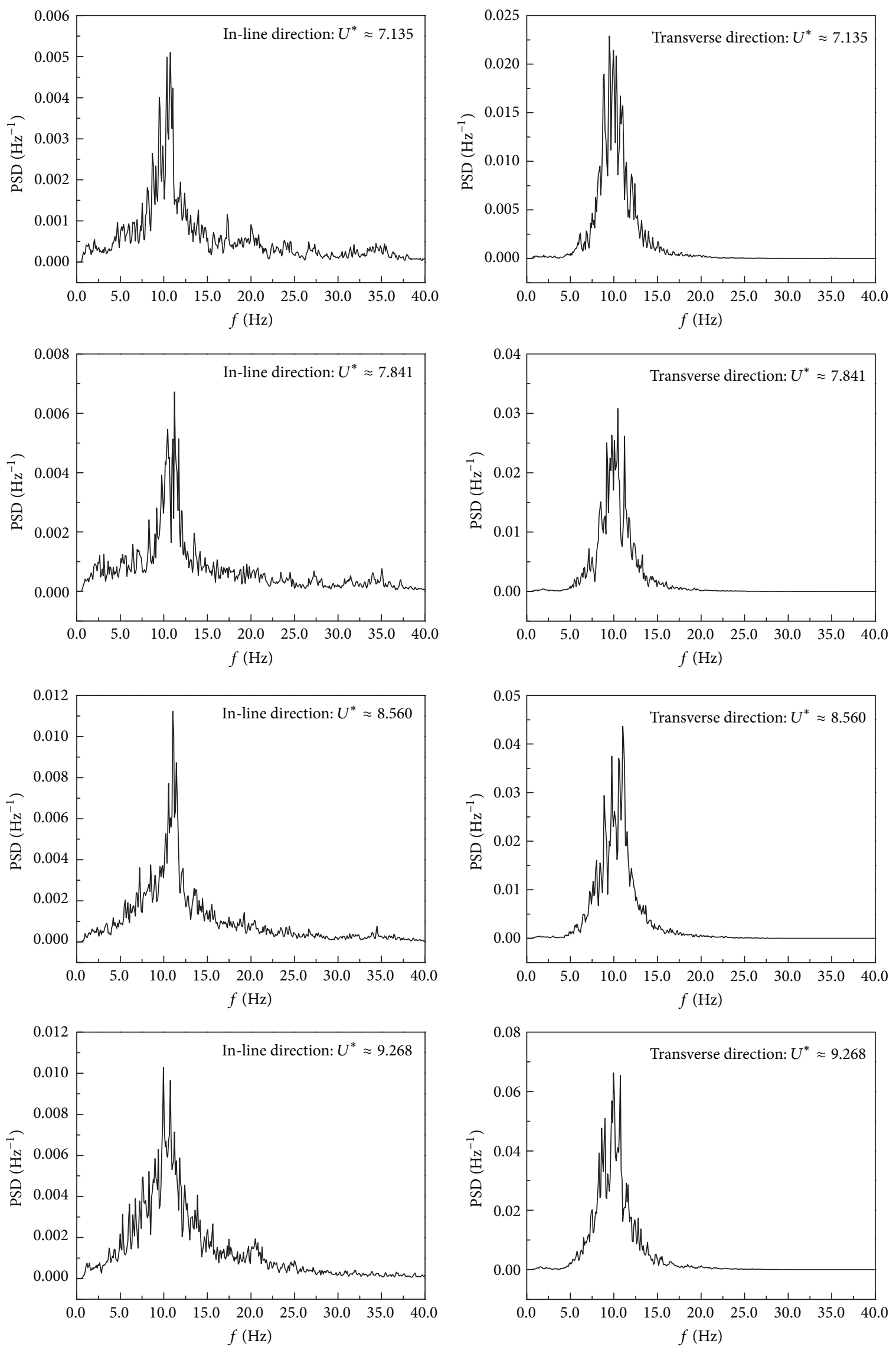

Figure 10: Continued. 

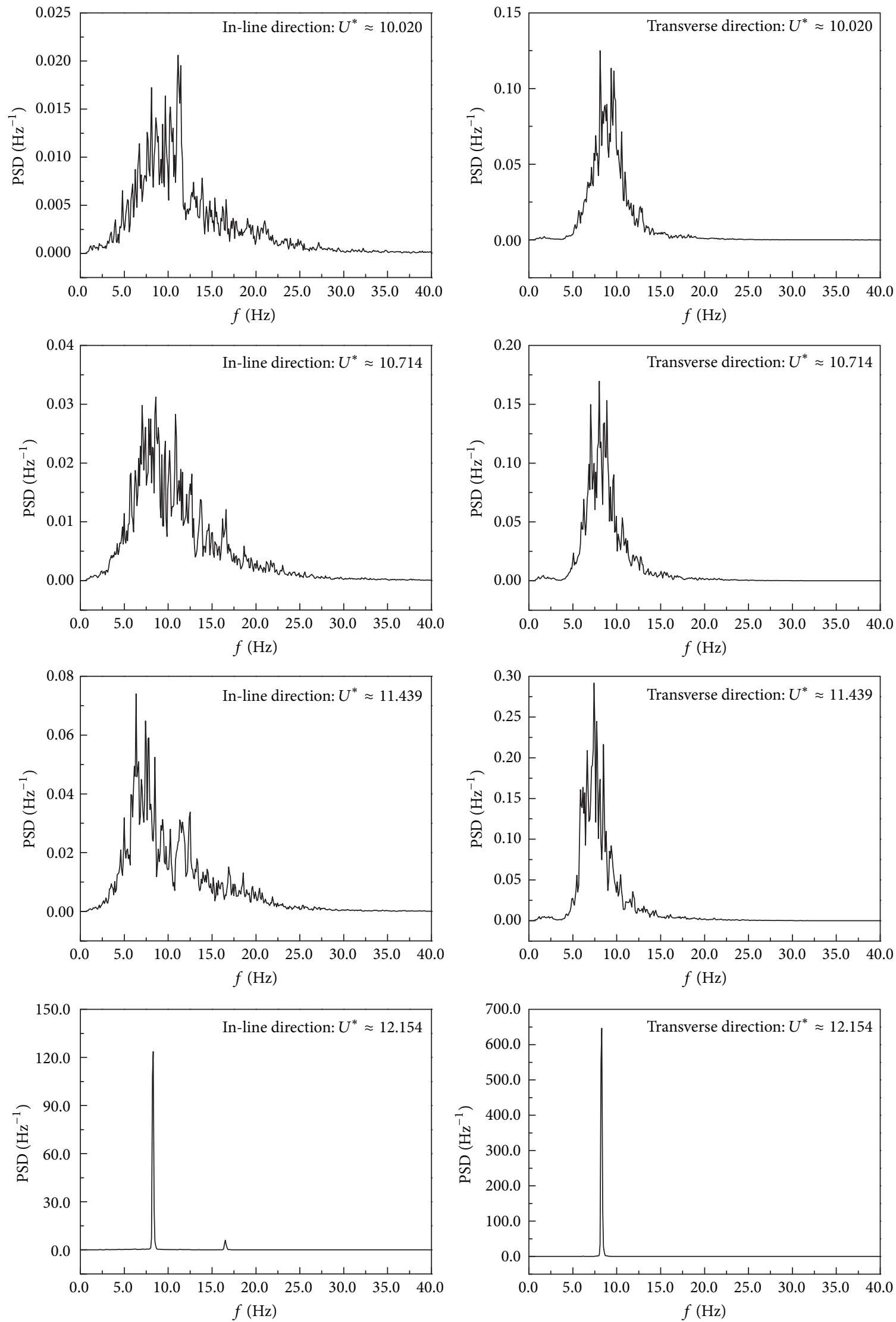

FIgURE 10: Power spectrum density functions of stainless steel tube vibrations with the increasing of flow velocity. 

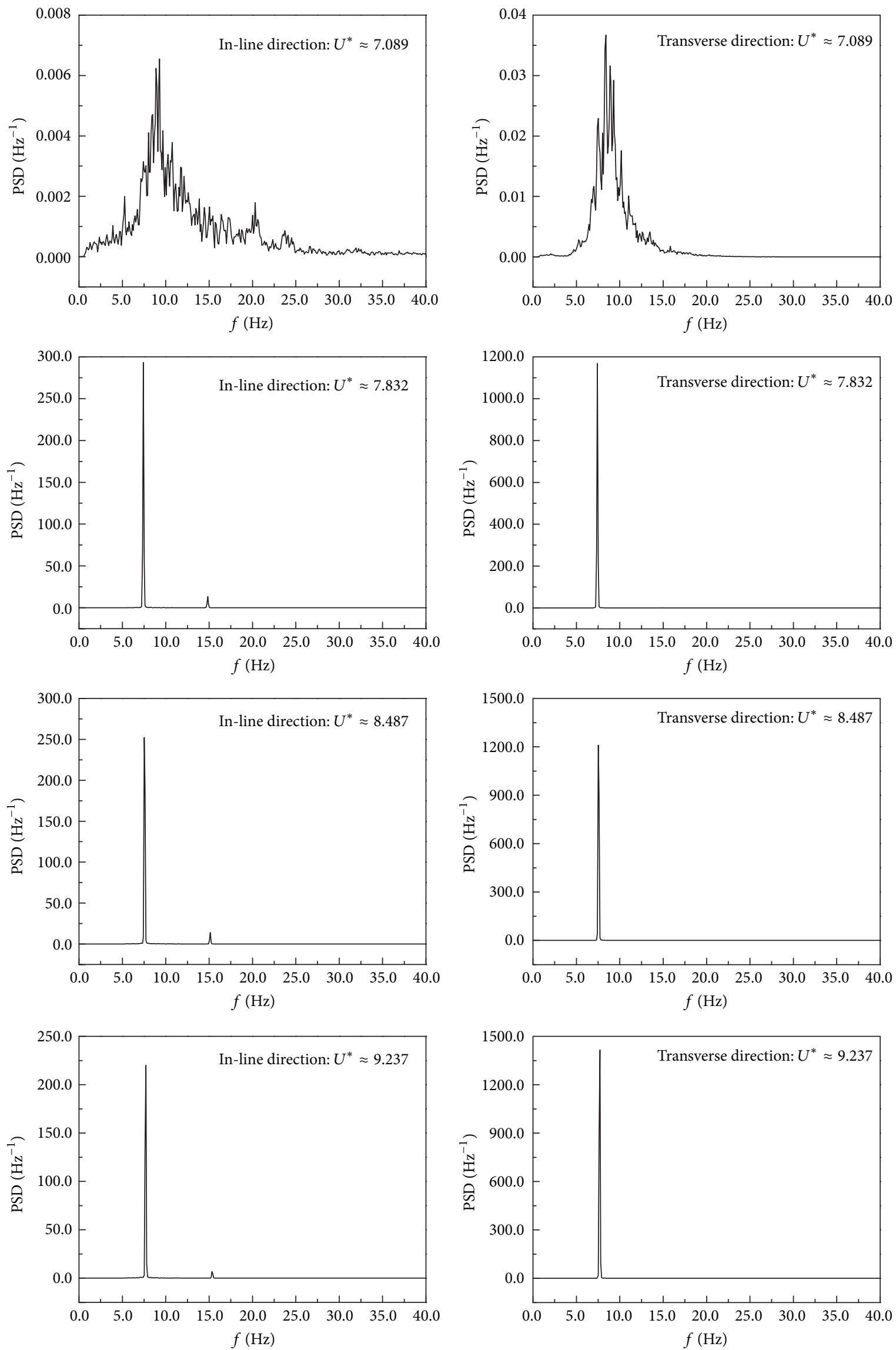

FIgUre 11: Continued. 

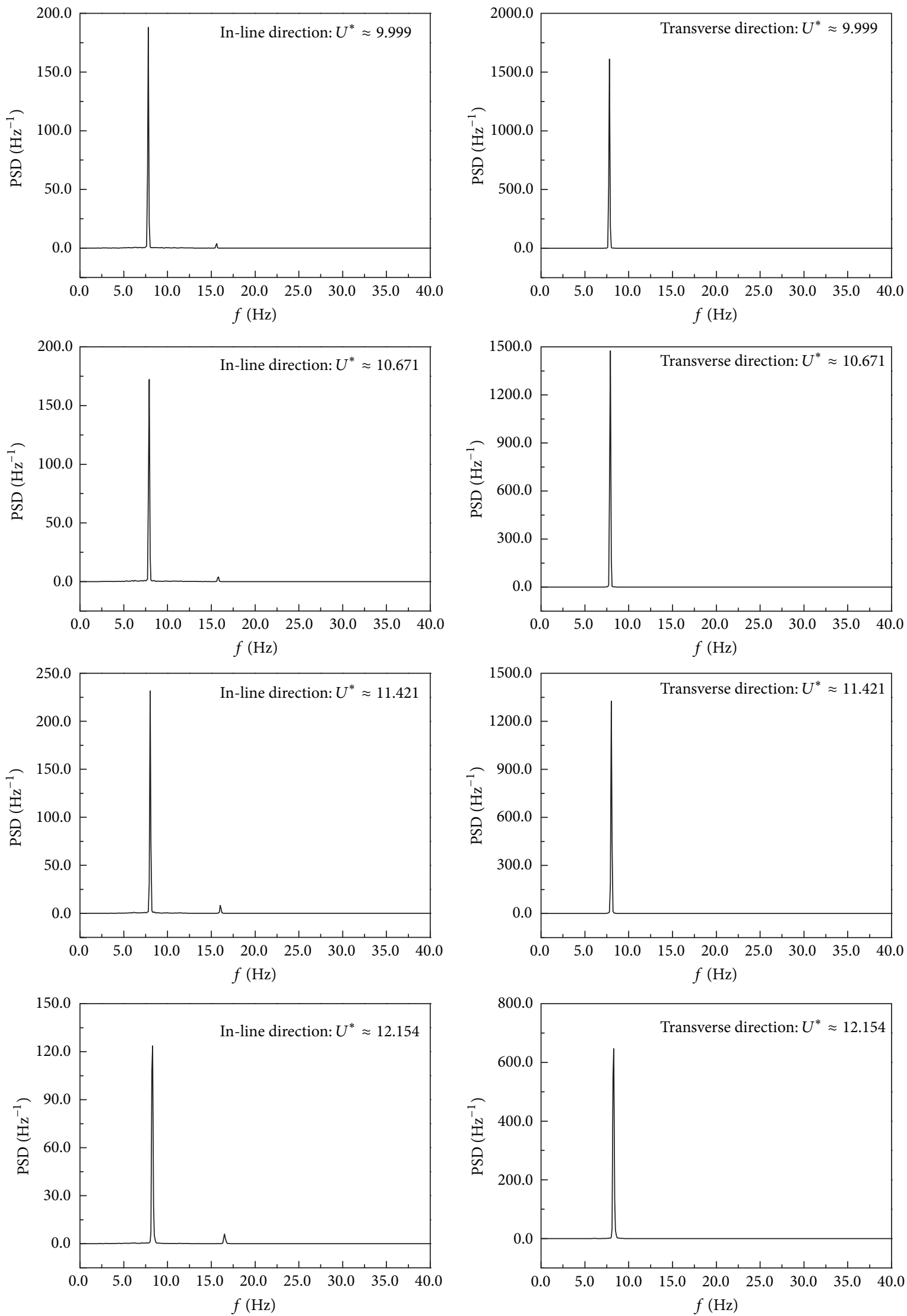

FIgURE 11: Power spectrum density functions of stainless steel tube vibrations with the decreasing of flow velocity. 

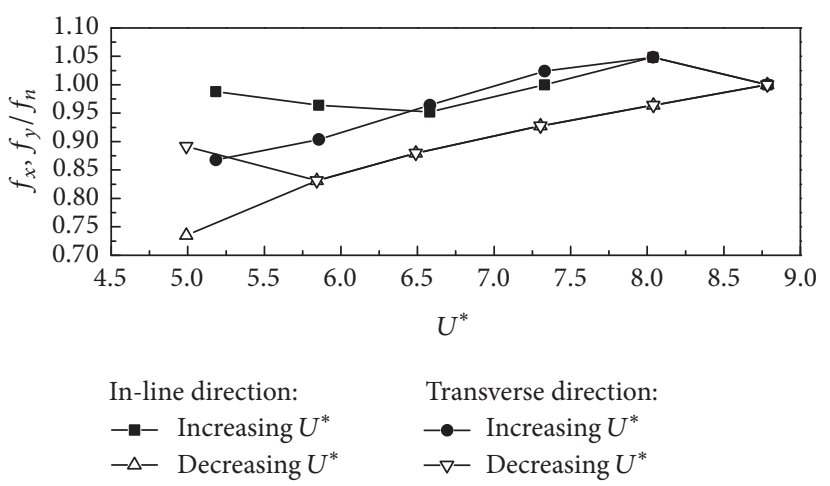

(a)

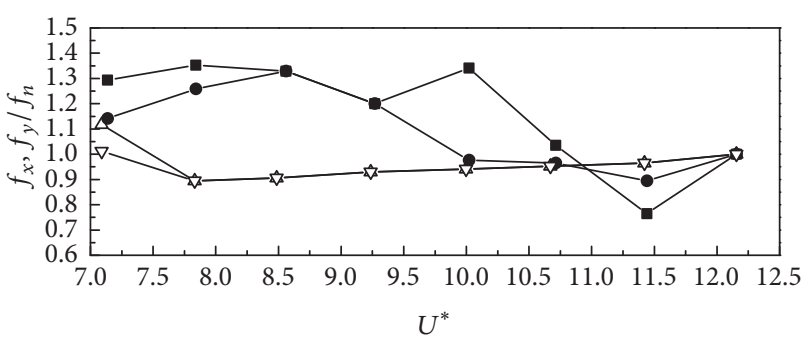

In-line direction: - - Increasing $U^{*}$ $-\triangle-$ Decreasing $U^{*}$

Transverse direction: $\rightarrow \bullet$ Increasing $U^{*}$ $-\nabla-$ Decreasing $U^{*}$

(b)

FIgURE 12: Response frequency versus flow velocity. (a) Aluminum tube and (b) stainless steel tube.

frequencies with some fluctuations, considering the fact that the monitored tubes are in the state of turbulent buffeting with a broadband frequency spectrum. The critical reduced pitch flow velocity of fluid-elastic instability of the monitored aluminum tube is 8.036, whereas that of the monitored stainless steel tube is 11.439. Meanwhile, as shown in Figure 7, fluid-elastic instability of the monitored aluminum tube occurred at $U^{*} \approx 8.785$, whereas that of the monitored stainless steel tube occurred at $U^{*} \approx 12.154$; these values are above their critical $U^{*}$ values. At this time, the dominant in-line and transverse frequencies of the monitored aluminum tube are all $8.105 \mathrm{~Hz}$, whereas those of the monitored stainless steel tube are all $8.301 \mathrm{~Hz}$. As a supplement, the frequency corresponding to the subharmonic peak in the power spectrum density function obtained from the monitored aluminum tube in-line vibration is $16.211 \mathrm{~Hz}$ and that from the monitored stainless steel tube in-line vibration is $16.504 \mathrm{~Hz}$. According to the figure plotted by Fitz-Hugh [36], the theoretical Strouhal number (St) is 0.5 for the two tube bundles in this experiment. Strouhal number is determined by the following equation:

$$
\mathrm{St}=\frac{f D}{U_{g}},
$$

where $f$ is the vortex shedding frequency in the tube bundle and $U_{g}$ is the mean gap velocity. For normal square and normal triangular bundles, $U_{g}$ is equal to $U_{p}$. However, for parallel triangular bundles, $U_{g}$ should be calculated as

$$
U_{g}=\frac{2 P \cos \alpha}{2 P \cos \alpha-D} U_{\infty}
$$

where $\alpha$ is equal to $30^{\circ}$ in this experiment. By applying the theoretical Strouhal number and $(8)$, at the point $U^{*} \approx 8.785$ in the monitored aluminum tube vibrations, the calculated vortex shedding frequencies are $21.788 \mathrm{~Hz}$, which is not equal to either $8.105 \mathrm{~Hz}$ or $16.211 \mathrm{~Hz}$. Similarly, at the point $U^{*} \approx 12.154$ in the monitored stainless steel tube vibrations, the calculated vortex shedding frequencies are $30.872 \mathrm{~Hz}$, which is not equal to either $8.301 \mathrm{~Hz}$ or $16.504 \mathrm{~Hz}$. Due to these mismatches, vortex shedding did not likely occur. The instability behaviors of both tubes were caused by fluid-elastic instability, because the vibration amplitudes increase rapidly once a critical crossflow velocity is exceeded, as shown in Figure 7.

No matter in the monitored aluminum tube vibrations or in the monitored stainless steel tube vibrations, the response frequency curves corresponding to decreasing $U^{*}$ all deviate from those corresponding to increasing $U^{*}$. As soon as $U^{*}$ values decreased to 4.991 for the monitored aluminum tube vibrations and 7.089 for the monitored stainless steel tube vibrations, the turbulent buffeting phenomenon, which is accompanied with a broadband frequency spectrum, occurred instead of fluid-elastic instability. In Figure 7, the large-amplitude vibrations of the monitored aluminum tube occur in the reduced pitch flow velocity range of 8.785 to 5.843 with decreasing flow velocity. According to (8) and (9), the calculated vortex shedding frequencies are in the range of $21.788 \mathrm{~Hz}$ to $14.492 \mathrm{~Hz}$. However, the actual dominant frequencies obtained from both in-line and transverse vibrations of the monitored aluminum tube in the reduced pitch flow velocity range of 8.785 to 5.843 with decreasing flow velocity are from $8.106 \mathrm{~Hz}$ to $6.738 \mathrm{~Hz}$. The calculated vortex shedding frequencies are very different from the actual dominant vibration frequencies in the case of aluminum tube. Similarly, the large-amplitude vibrations of the monitored stainless steel tube occur in the reduced pitch flow velocity range of 12.154 to 7.833 with decreasing flow velocity. According to (8) and (9), the calculated vortex shedding frequencies are in the range of $30.872 \mathrm{~Hz}$ to $19.895 \mathrm{~Hz}$. However, the actual dominant frequencies obtained from both in-line and transverse vibrations of the monitored stainless steel tube in the reduced pitch flow velocity range of 12.154 to 7.833 with decreasing flow velocity are from $8.301 \mathrm{~Hz}$ to $7.422 \mathrm{~Hz}$. The calculated vortex shedding frequencies are very different from the actual dominant vibration frequencies in the case of stainless steel tube as well. The abovementioned analyses confirmed that vortex shedding did not occur during the experiments. With $U^{*}$ ranging from $U^{*} \approx 8.785$ to $U^{*} \approx$ 5.843 in the aluminum tube vibrations and from $U^{*} \approx 12.154$ to $U^{*} \approx 7.832$ in the stainless steel tube vibrations, the 
TABLE 2: Summary of fluid-elastic instability results.

\begin{tabular}{lcccc}
\hline & \multicolumn{3}{c}{ Increasing $U^{*}$} & \multicolumn{2}{c}{ Decreasing $U^{*}$} \\
& In-line direction & Transverse direction & In-line direction & Transverse direction \\
\hline Aluminum tube & & & & \\
$U_{P C}(\mathrm{~m} / \mathrm{s})$ & 0.782 & 0.782 & 0.485 & 0.485 \\
$U_{P C} / f_{n} D$ & 8.036 & 8.036 & 4.991 & 4.991 \\
$2 \pi \zeta \mathrm{m} / \rho D^{2}$ & 0.832 & 0.377 & 1.023 & 0.328 \\
$K$ calculated for this criterion & 8.810 & 13.088 & 4.935 & 8.715 \\
\hline Stainless steel tube & & & & \\
$U_{P C}(\mathrm{~m} / \mathrm{s})$ & 1.140 & 1.140 & 0.706 & 0.706 \\
$U_{P C} / f_{n} D$ & 11.439 & 11.439 & 7.089 & 7.089 \\
$2 \pi \zeta \mathrm{m} / \rho D^{2}$ & 0.923 & 0.617 & 0.456 & 0.544 \\
$K$ calculated for this criterion & 11.907 & 14.563 & 10.498 & 9.611 \\
\hline
\end{tabular}

monitored tubes were in the state of fluid-elastic instability. The response frequencies of both tubes decreased, while the response frequencies of the aluminum tube vibrations decreased much more than those of the stainless steel tube vibrations. This result may be due to the fact that the mass ratio of the stainless steel tube is larger than that of the aluminum tube, so the inertia effect of the stainless steel tube is much more significant than that of the aluminum tube.

3.4. Added Mass Coefficient. The natural frequencies of structures oscillating in flow are affected by the inertia of the fluid accelerated by the structures. The inertia of the fluid accelerated by the structures is treated as added mass, $m_{a}$, which can be calculated by (2) in Section 2.1.

For right circular tubes in still fluid, the theoretical added mass coefficient is equal to 1 [34]. However, when the tube is vibrating in a confined space, the effective added mass coefficient is larger than 1 . The added mass coefficient in flow can be calculated as follows [37]:

$$
c_{a}=\left(\frac{\gamma_{c}}{\gamma_{w}}\right)\left[\left(\frac{f_{a}}{f_{n}}\right)^{2}-1\right],
$$

where $f_{a}$ is the natural frequency of a tube in vacuum (usually taken in air), $f_{n}$ is the natural frequency of a tube in flow, and $\gamma_{c}$ and $\gamma_{w}$ are the tube and fluid specific gravities, respectively. The density of the water used in experiments was $998.200 \mathrm{~kg} / \mathrm{m}^{3}$. The density of the water in the standard state (one normal atmospheric pressure and $3.98^{\circ} \mathrm{C}$ ) was $999.972 \mathrm{~kg} / \mathrm{m}^{3}$. The specific gravity of the water used in experiments was $998.200 / 999.972=0.9982$. The density of the aluminum tube was calculated as follows:

$$
\frac{0.02213}{(\pi / 4) \times 0.012 \times 0.012 \times 0.130}=1505.170 \mathrm{~kg} / \mathrm{m}^{3} .
$$

Similarly, the density of the stainless steel tube was calculated using the following equation:

$$
\frac{0.04619}{(\pi / 4) \times 0.012 \times 0.012 \times 0.130}=3141.610 \mathrm{~kg} / \mathrm{m}^{3} .
$$

As a result, the specific gravity of the aluminum tube was $1505.170 / 999.972=1.5052$ and that of the stainless steel tube was $3141.610 / 999.972=3.1417$.
The added mass coefficients in the in-line and transverse vibrations corresponding to both tubes in the state of fluidelastic instability are summarized and plotted in Figure 13. For both tube vibrations, the curves corresponding to the in-line direction are similar to those corresponding to the transverse direction. It is caused by that the dominant frequencies of the in-line and transverse vibrations obtained from the $U^{*}$ ranges in which the monitored tube was in the state of fluid-elastic instability were same. All added mass coefficients are larger than 1, resulting from the relative motions of the monitored tubes in the rigid bundles.

3.5. Fluid-Elastic Instability Analysis. The criterion for fluidelastic instability, proposed by Pettigrew and Gorman [9], was applied in data analyses. The critical points confirmed in Figure 7 are all plotted in Figure 14, and they all lay in the unstable region. This finding indicates that the largeamplitude vibrations of the monitored aluminum tube and stainless steel tube were caused by fluid-elastic instability again. Table 2 presents a summary of the fluid-elastic instability results. Corresponding dimensionless parameters are also given. The value of each $K$ shown in Table 2 was calculated directly by putting the values of related $U_{P C} / f_{n} D$ and $2 \pi \zeta \mathrm{m} / \rho D^{2}$ obtained at the critical pitch flow velocity into the equation of $U_{P C} / f_{n} D=K\left(2 \pi \zeta m / \rho D^{2}\right)^{0.5}$. This method was referenced from Mureithi et al. [38]. The calculated $K$ values are all larger than the literature values shown in Figure 14, considering the fact that only one tube was mounted elastically in the rigid tube bundle in the cases of both tube bundles.

\section{Conclusions}

In this paper, two parallel triangular tube bundles with the same $P / D$ value of 1.67 and different mass ratio values were tested for fluid-elastic instability, respectively. Only one tube was mounted elastically in both the aluminum and stainless steel tube bundles. The following conclusions were drawn from the present work.

(1) No matter in the aluminum tube bundle or the stainless steel tube bundle, fluid-elastic instability 

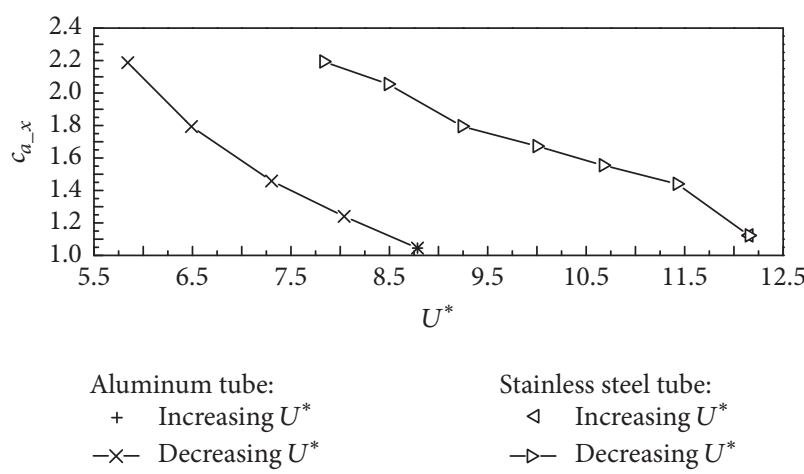

(a)

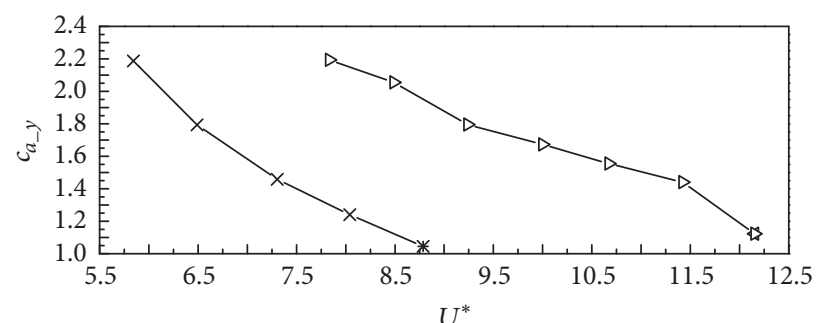

Aluminum tube:

+ Increasing $U^{*}$

$-\times-$ Decreasing $U^{*}$
Stainless steel tube:

$\triangleleft$ Increasing $U^{*}$

$\rightarrow-$ Decreasing $U^{*}$

(b)

FIgURE 13: Added mass coefficients. (a) In-line direction; (b) transverse direction.

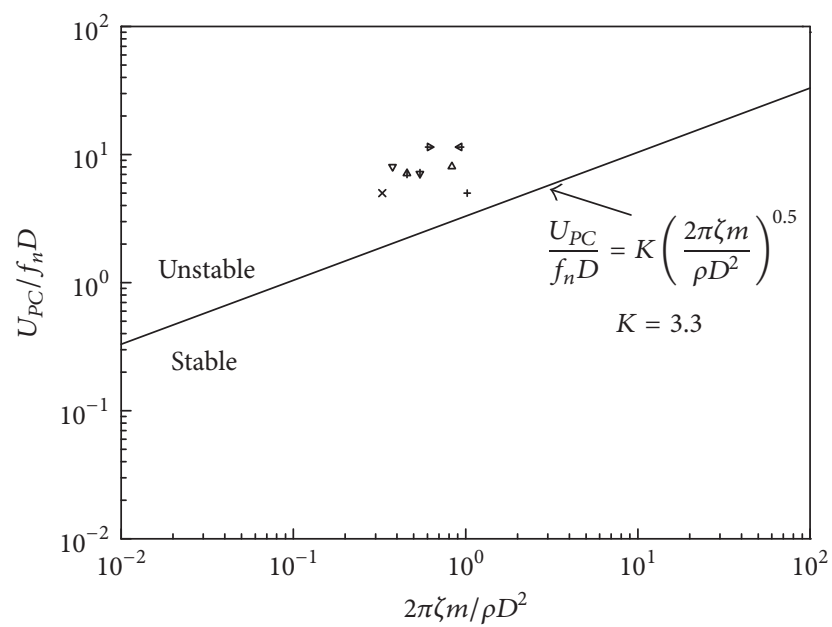

$\begin{array}{lc}\text { Aluminum: } & \text { Stainless steel: } \\ \text { Increasing } U^{*} & \text { Increasing } U^{*} \\ \Delta \text { In-line direction } & \star \text { In-line direction } \\ \quad \nabla \text { Transverse direction } & \rightarrow \text { Transverse direction } \\ \text { Decreasing } U^{*} & \text { Decreasing } U^{*} \\ \quad+\text { In-line direction } & \star \text { In-line direction } \\ \quad \times \text { Transverse direction } & \star \text { Transverse direction }\end{array}$

FIGURE 14: Fluid-elastic instability map for the two parallel triangular tube bundles.

occurred in both the in-line and transverse vibrations. Moreover, the response amplitudes obtained from the transverse vibrations were larger than those obtained from the in-line vibrations.

(2) Nonlinear hysteresis occurred in both the aluminum tube bundle vibrations and the stainless steel tube bundle vibrations.

(3) When the flow velocity is decreasing, the tubes which have been in the state of fluid-elastic instability can keep on this state for a certain flow velocity range. During this process, the response frequencies of the tubes will decrease. Furthermore, the response frequencies of the aluminum tube can decrease much more than those of the stainless steel tube.
(4) The added mass coefficients corresponding to fluidelastic instability were obtained for both tubes. They were all larger than 1.

(5) The fluid-elastic instability criterion proposed by Pettigrew and Gorman [9] was applied. The critical points for both tube vibrations all lay in the unstable region. The calculated fluid-elastic instability constants, which are consistent with these experiments, are all larger than the theoretical value.

\section{Nomenclature}

$a$ : Length of the cross section of the test section, $m$

$b$ : Width of the cross section of the test section, $m$

$c_{a}: \quad$ Added mass coefficient

$c_{a-x}$ : Added mass coefficient in the in-line direction

$c_{a_{-} y}:$ Added mass coefficient in the transverse direction

$D: \quad$ Outer diameter of the tube, $m$

$f_{a}$ : Natural frequency in vacuum (usually taken in air) in the in-line or the transverse direction, $\mathrm{Hz}$

$f_{n}$ : Natural frequency in water in the in-line or the transverse direction, $\mathrm{Hz}$

$f_{\text {na } x}$ : Natural frequency in air in the in-line direction, $\mathrm{Hz}$

$f_{n a_{-}-}$: Natural frequency in air in the transverse direction, $\mathrm{Hz}$

$f_{n w-x}$ : Natural frequency in water in the in-line direction, $\mathrm{Hz}$

$f_{n w_{-} y}$ : Natural frequency in water in the transverse direction, $\mathrm{Hz}$

$K$ : Constant of the criterion equation of fluid-elastic instability

$L: \quad$ The length of the tube, $\mathrm{m}$

M: $\quad$ Structural mass (including acceleration transducers inside), $\mathrm{kg}$

$m^{*}: \quad$ Mass ratio 
$m_{a}:$ Added mass, $\mathrm{kg}$

$m_{d}$ : The mass of the displaced fluid, $\mathrm{kg}$

$m_{t}$ : The oscillating mass of the system, $\mathrm{kg}$

$P$ : $\quad$ Tube center-to-center spacing of every two nearest tubes, $\mathrm{m}$

$P / D$ : Pitch-to-diameter ratio

$U_{g}:$ Mean gap flow velocity, $\mathrm{m} / \mathrm{s}$

$U_{p}$ : Pitch flow velocity, $\mathrm{m} / \mathrm{s}$

$U_{\infty}$ : Freestream velocity, $\mathrm{m} / \mathrm{s}$

$U^{*}$ : Reduced pitch flow velocity

$V: \quad$ Flow rate, $\mathrm{m}^{3} / \mathrm{h}$.

\section{Greek Letters}

$\alpha$ : Bundle pitch angle, ${ }^{\circ}$

$\gamma_{c}$ : The tube specific gravity

$\gamma_{w}$ : The fluid specific gravity

$\xi_{x}$ : Damping ratio in flow

$\xi_{x a}$ : Damping ratio in air in the in-line direction without flow

$\xi_{y a}:$ Damping ratio in air in the transverse direction without flow

$\xi_{x w}$ : Damping ratio in water in the in-line direction without flow

$\xi_{y w}$ : Damping ratio in water in the transverse direction without flow

$\rho$ : The density of the fluid.

\section{Superscript}

*: Nondimensional.

\section{Subscript}

n: Natural

$P$ : Tube center-to-center spacing of every two nearest tubes, $\mathrm{m}$

$\infty$ : Freestream

$x$ : In-line direction

$y$ : Transverse direction.

\section{Competing Interests}

The authors declare that there is no conflict of interests regarding the publication of this paper.

\section{Acknowledgments}

This research was supported financially by International S\&T Cooperation Program of China, ISTCP (no. 2015DFR40910).

\section{References}

[1] P. Plaschko, "Deterministic and stochastic oscillations of a flexible cylinder in arrays of static tubes," Nonlinear Dynamics, vol. 30, no. 4, pp. 337-355, 2002.

[2] M. Tang, Q. Ni, Y. Luo, Y. Wang, and L. Wang, "Flow-induced vibration of curved cylinder arrays subject to loose support," Nonlinear Dynamics, vol. 78, no. 4, pp. 2533-2545, 2014.
[3] H. X. He and J. W. Li, "Study on the effect and mechanism of aerodynamic measures for the vortex-induced vibration of separate pairs of box girders in cable-stayed bridges," Shock and Vibration, vol. 2015, Article ID 792957, 11 pages, 2015.

[4] M. T. Song, D. Q. Cao, and W. D. Zhu, "Vortex-induced vibration of a cable-stayed bridge," Shock and Vibration, vol. 2016, Article ID 1928086, 14 pages, 2016.

[5] S. S. Chen, "Instability mechanisms and stability criteria of a group of circular cylinders subjected to cross-flow-part i: theory," Journal of Vibration, Acoustics, Stress, and Reliability in Design, vol. 105, no. 1, pp. 51-58, 1983.

[6] S. S. Chen, "Instability mechanisms and stability criteria of a group of circular cylinders subjected to cross-flow," Journal of Vibration, Acoustics, Stress, and Reliability in Design, vol. 105, no. 2, pp. 253-260, 1983.

[7] M. P. Paidoussis and S. J. Price, "Mechanisms underlying flowinduced instabilities of cylinder arrays in crossflow," Journal of Fluid Mechanics, vol. 187, pp. 45-59, 1988.

[8] H. J. Connors, An experimental investigation of the flow-induced vibration of tube arrays in cross flow [Ph.D. thesis], University of Pittsburgh, 1969.

[9] M. J. Pettigrew and D. J. Gorman, "Vibration of heat exchanger components in liquid and two-phase cross flow," in Proceedings of the B.N.E.S. Conference on Vibration in Nuclear Plant, Keswick, U.K, 1978.

[10] M. P. Païdoussis, "Fluidelastic vibration of cylinder arrays in axial and cross flow: state of the art," Journal of Sound and Vibration, vol. 76, no. 3, pp. 329-360, 1981.

[11] D. S. Weaver and J. A. Fitzpatrick, "A review of flow-induced vibration in heat-exchangers," in Proceedings of the International Conference on Flow-Induced Vibration, Bowness-onWindermere, UK, 1987.

[12] P. Piteau, X. Delaune, J. Antunes, and L. Borsoi, "Experiments and computations of a loosely supported tube in a rigid bundle subjected to single-phase flow," Journal of Fluids and Structures, vol. 28, pp. 56-71, 2012.

[13] K. Yan, P.-Q. Ge, and J. Hong, "Experimental study of shell side flow-induced vibration of conical spiral tube bundle," Journal of Hydrodynamics, vol. 25, no. 5, pp. 695-701, 2013.

[14] B. Anderson, M. Hassan, and A. Mohany, "Modelling of fluidelastic instability in a square inline tube array including the boundary layer effect," Journal of Fluids and Structures, vol. 48, pp. 362-375, 2014.

[15] S. El Bouzidi and M. Hassan, "An investigation of time lag causing fluidelastic instability in tube arrays," Journal of Fluids and Structures, vol. 57, pp. 264-276, 2015.

[16] T.-K. Lin and M.-H. Yu, "An experimental study on the cross-flow vibration of a flexible cylinder in cylinder arrays," Experimental Thermal and Fluid Science, vol. 29, no. 4, pp. 523536, 2005.

[17] T. Sasakawa, A. Serizawa, and Z. Kawara, "Fluid-elastic vibration in two-phase cross flow," Experimental Thermal and Fluid Science, vol. 29, no. 3, pp. 403-413, 2005.

[18] S. Umeda and W.-J. Yang, "Interaction of von Karman vortices and intersecting main streams in staggered tube bundles," Experiments in Fluids, vol. 26, no. 5, pp. 389-396, 1999.

[19] R. M. C. So, Y. Zhou, and M. H. Liu, "Free vibrations of an elastic cylinder in a cross flow and their effects on the near wake," Experiments in Fluids, vol. 29, no. 2, pp. 130-144, 2000.

[20] H. D. AkaydIn, N. Elvin, and Y. Andreopoulos, "Wake of a cylinder: a paradigm for energy harvesting with piezoelectric 
materials," Experiments in Fluids, vol. 49, no. 1, pp. 291-304, 2010.

[21] F. Gu, J. S. Wang, Z. M. Zhao, J. Sui, and X. Q. Qiao, "Wind and water tunnel experiments on flow control for a circular cylinder with axial-rod shrouds attached," Chinese Journal of Hydrodynamics Series A, vol. 27, no. 3, pp. 293-302, 2012.

[22] J. H. Lever and D. S. Weaver, "On the stability of heat exchanger tube bundles, part II: numerical results and comparison with experiments," Journal of Sound and Vibration, vol. 107, no. 3, pp. 393-410, 1986.

[23] J. H. Lever and D. S. Weaver, "On the stability of heat exchanger tube bundles, part I: modified theoretical model," Journal of Sound and Vibration, vol. 107, no. 3, pp. 375-392, 1986.

[24] S. J. Price and M. P. Païdoussis, "The flow-induced response of a single flexible cylinder in an in-line array of rigid cylinders," Journal of Fluids and Structures, vol. 3, no. 1, pp. 61-82, 1989.

[25] J. Marn and I. Catton, "On stability analysis of a flexible cylinder in an array of rigid cylinders," Transactions of the ASME. Journal of Fluids Engineering, vol. 114, no. 1, pp. 12-19, 1992.

[26] G. Harran, "Influence of the mass ratio on the fluidelastic instability of a flexible cylinder in a bundle of rigid tubes," Journal of Fluids and Structures, vol. 47, pp. 71-85, 2014.

[27] Y. Joo and V. K. Dhir, "An experimental study of drag on a single tube and on a tube in an array under two-phase cross flow," International Journal of Multiphase Flow, vol. 20, no. 6, pp.10091019, 1994.

[28] D. R. Mitra, Fluid-Elastic Instability in Tube Arrays Subjected to Air-Water and Steam-Water Cross-Flow, University of California, Los Angeles, Calif, USA, 2005.

[29] N. Cagney and S. Balabani, "Wake modes of a cylinder undergoing free streamwise vortex-induced vibrations," Journal of Fluids and Structures, vol. 38, pp. 127-145, 2013.

[30] F. Hara, "Vibration of a single row of circle cylinders subjected to two-phase bubble cross-flow," in Proceedings of the International Conference on Flow Induced Vibrations, Bowness-onWindermere, UK, 1987.

[31] H. Marcollo and J. B. Hinwood, "On shear flow single mode lock-in with both cross-flow and in-line lock-in mechanisms," Journal of Fluids and Structures, vol. 22, no. 2, pp. 197-211, 2006.

[32] S. Huang, M. Khorasanchi, and K. Herford, "Drag amplification of long flexible riser models undergoing multi-mode VIV in uniform currents," Journal of Fluids and Structures, vol. 27, no. 3, pp. 342-353, 2011.

[33] R. D. Blevins, Flow-Induced Vibration, Van Nostrand Reinhold, New York, NY, USA, 1st edition, 1977.

[34] A. Khalak and C. H. K. Williamson, "Motions, forces and mode transitions in vortex-induced vibrations at low mass-damping," Journal of Fluids and Structures, vol. 13, no. 7-8, pp. 813-851, 1999.

[35] G. R. Franzini, R. T. Gonçalves, J. R. Meneghini, and A. L. C. Fujarra, "One and two degrees-of-freedom vortex-induced vibration experiments with yawed cylinders," Journal of Fluids and Structures, vol. 42, pp. 401-420, 2013.

[36] J. S. Fitz-Hugh, Flow Induced Vibration in Heat Exchangers, Atomic Energy Research Establishment, 1973.

[37] D. S. Weaver and H. C. Yeung, "The effect of tube mass on the flow induced response of various tube arrays in water," Journal of Sound and Vibration, vol. 93, no. 3, pp. 409-425, 1984.

[38] N. W. Mureithi, C. Zhang, M. Ruël, and M. J. Pettigrew, "Fluidelastic instability tests on an array of tubes preferentially flexible in the flow direction," Journal of Fluids and Structures, vol. 21, no. 1, pp. 75-87, 2005. 


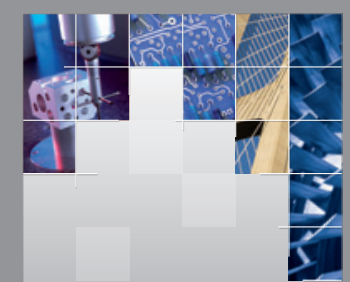

\section{Enfincering}
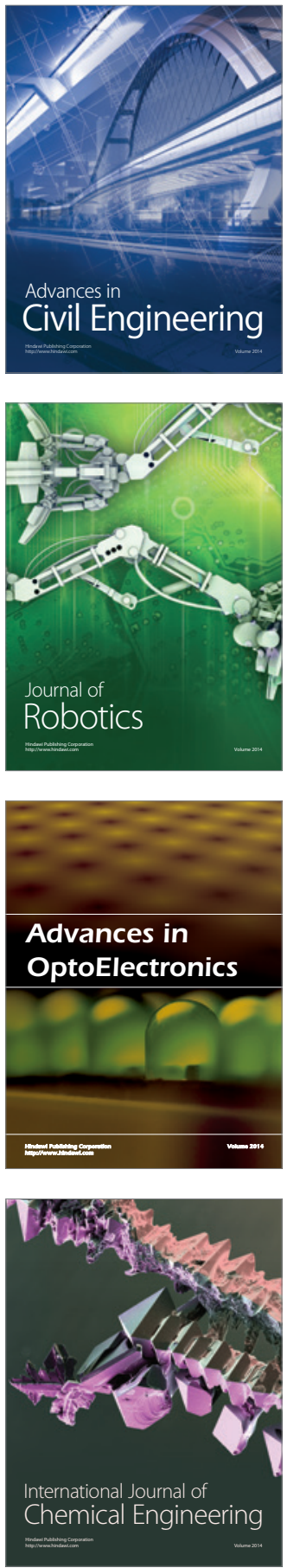

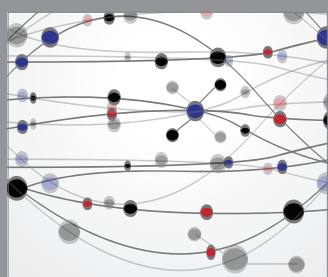

The Scientific World Journal

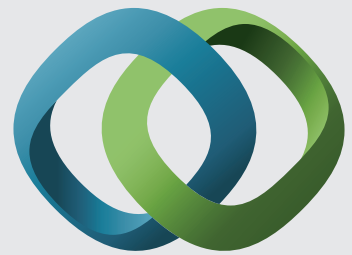

\section{Hindawi}

Submit your manuscripts at

http://www.hindawi.com
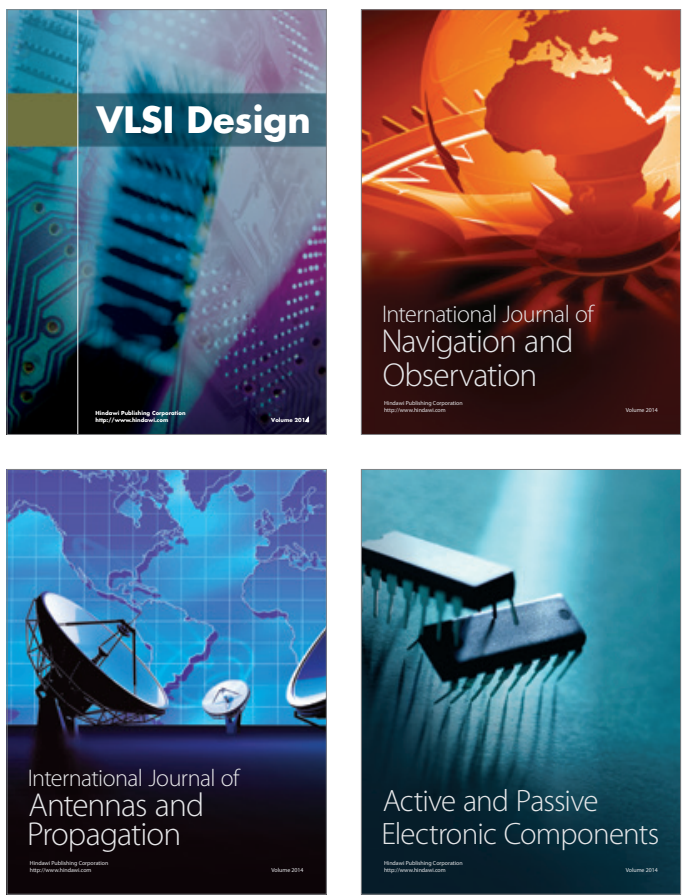
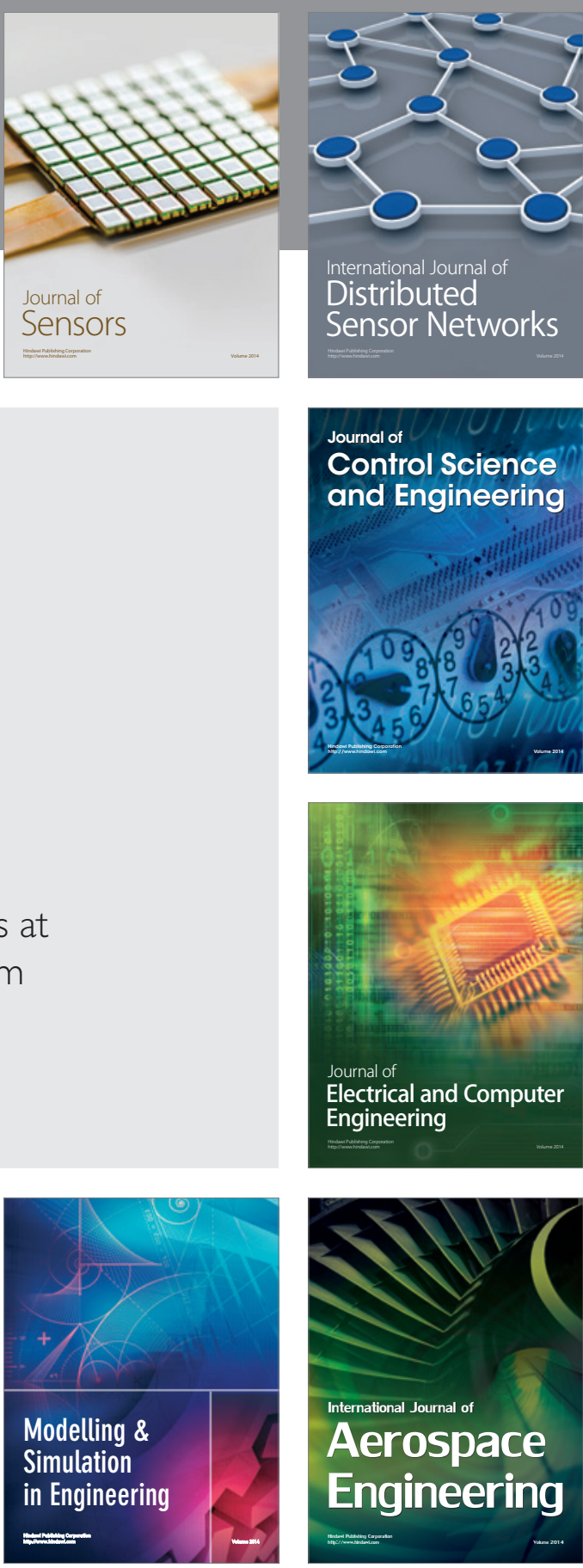

International Journal of

Distributed

Sensor Networks

Journal of

Control Science

and Engineering
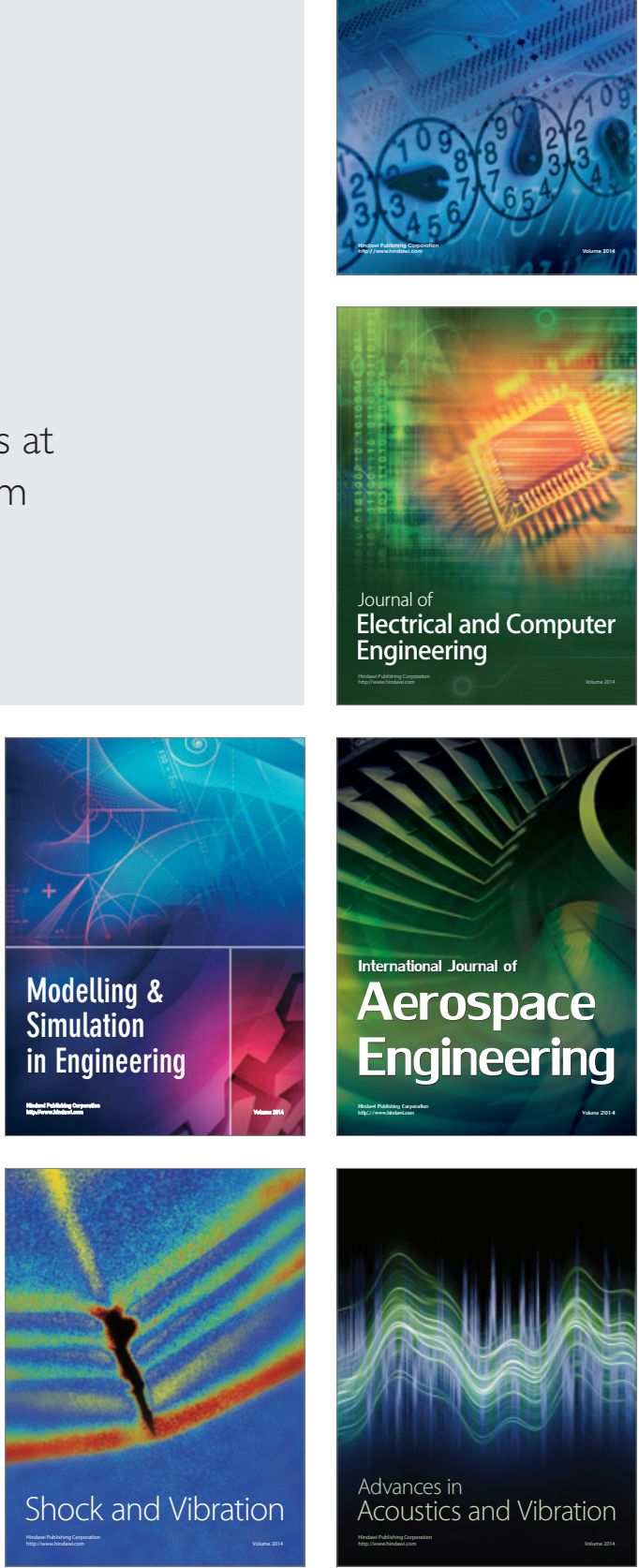